\title{
Thioredoxin activity confers resistance against oxidative stress in tumor-infiltrating NK cells
}

\author{
Ying Yang,,${ }^{1,2}$ Shi Yong Neo, ${ }^{2}$ Ziqing Chen, ${ }^{2}$ Weiyingqi Cui, ${ }^{2}$ Yi Chen, ${ }^{2}$ Min Guo, ${ }^{2}$ Yongfang Wang, ${ }^{3}$ Haiyan $\mathrm{Xu},{ }^{3}$ Annina Kurzay, ${ }^{2,4}$ \\ Evren Alici, ${ }^{5}$ Lars Holmgren, ${ }^{2}$ Felix Haglund, ${ }^{2}$ Kai Wang, ${ }^{1}$ and Andreas Lundqvist ${ }^{2}$ \\ 'School of Medicine, Zhejiang University, Hangzhou, China. ${ }^{2}$ Department of Oncology-Pathology, Karolinska Institutet, Stockholm, Sweden. ${ }^{3}$ Second Affiliated Hospital, Zhejiang University School of \\ Medicine, Hangzhou, China. ${ }^{4}$ German Cancer Research Center, DKFZ, Heidelberg, Germany. ${ }^{5}$ Department of Medicine Huddinge, Karolinska Institutet, Stockholm, Sweden.
}

\begin{abstract}
To improve the clinical outcome of adoptive NK cell therapy in patients with solid tumors, NK cells need to persist within the tumor microenvironment (TME) in which the abundance of ROS could dampen antitumor immune responses. In the present study, we demonstrated that IL-15-primed NK cells acquired resistance against oxidative stress through the thioredoxin system activated by mTOR. Mechanistically, the activation of thioredoxin showed dependence on localization of thioredoxininteracting protein. We show that NK cells residing in the tumor core expressed higher thiol densities that could aid in protecting other lymphocytes against ROS within the TME. Furthermore, the prognostic value of IL15 and the NK cell gene signature in tumors may be influenced by tobacco smoking history in patients with non-small-cell lung cancer (NSCLC). Collectively, the levels of reducing antioxidants in NK cells may not only predict better tumor penetrance but potentially even the immune therapy response.
\end{abstract}

\section{Introduction}

ROS are a diverse class of radical species that have different roles depending on their concentration. Considering their immunesuppressive effects, ROS have been proposed as a metabolic immune checkpoint within the tumor microenvironment (TME) (1-3). ROS are produced not only by stressed and highly metabolic tumor cells but also by activated immune cells such as granulocytes, macrophages, and myeloid-derived suppressor cells. Although oxidative stress is a double-edged sword in cancer biology, targeting the antioxidant pathway may represent a good strategy in combination with immunotherapy (4). Antioxidants such as reducing enzymes provide tumor cells with resistance against high oxidative stress, but on the contrary, the TME may potentially be reshaped with increasing immune infiltration and persistence of cytotoxic lymphocytes $(5,6)$. Thioredoxin is one of the main antioxidants in the cellular redox system that functions as a scavenger for ROS, with its primary function being to reduce oxidized cysteine residues and cleave disulfide bonds $(7,8)$. Elevated levels of thioredoxin often correlate with immune activation, regulating the survival of immune cells (9-11).

Since tobacco smoking is one of the major causes of lung cancer and contributes to the accumulation of oxidative stress in the lung TME $(12,13)$, lung cancer represents one of the more relevant cancer types in which to study the impact of ROS on immunity (4).

\section{Related Commentary: p. 5115}

Authorship note: YY and SYN are co-first authors.

Conflict of interest: The authors have declared that no conflict of interest exists. Copyright: (c) 2020, American Society for Clinical Investigation.

Submitted: February 26, 2020; Accepted: July 9, 2020; Published: September 14, 2020 Reference information: / Clin Invest. 2020;130(10):5508-5522.

https://doi.org/10.1172/JCI137585.
Tobacco smoking by itself not only contains high concentrations of ROS but also triggers inflammation, activating immune cells to upregulate ROS production (14). However, tobacco smoking may negatively affect the survival of lymphocytes residing in the lungs and hence modulate the tissue's immune landscape. A hallmark study also highlighted that the smoking signature influences tumor mutational burden and sensitivity to PD-1 blockade in nonsmall-cell lung cancer (NSCLC) (15). Smoking history may indeed influence a patient's clinical response to immune therapy, even though the dogma still remains that tobacco smoking promotes lung cancer progression.

NK cells are cytotoxic lymphocytes that contribute to immune surveillance against viral infections and cancer. Unlike conventional $\mathrm{CD}^{+} \mathrm{T}$ cells, the cytotoxicity functions of NK cells are robustly regulated by a repertoire of activating and inhibitory receptors. One of the key inhibitory ligands to provide a "don't kill" signal is MHC class I, which is often downregulated, resulting in increased sensitivity to NK cell-mediated cytotoxicity within the tumor (16-18). Existing therapeutic approaches to harness NK cells for cancer immunotherapy include adoptive cell therapy and use of inhibitors against NK cell checkpoints. However, adoptive NK cell therapy has so far only proven beneficial against hematological cancers, given its poor penetrance into solid tumors and inadequate cytotoxicity to tumor cells (19). Moreover, the function and fate of these NK cells are prone to be strongly influenced by the highly dynamic TME, considering that tissue-infiltrating NK cells may not be merely "killers" but can undergo a phenotypic switch to acquire other noncanonical functions (20-22).

Our previous study demonstrated that modulation of $\mathrm{NK}$ cells with IL-15 for adoptive cell therapy enhances effector functions regulated by STAT5 signaling and mTOR activation (23). Given that oxidative stress has profound immune-suppressive effects on NK cells (1-3), we sought to determine whether IL-15 
and mTOR activation of NK cells would confer resistance against oxidative stress. Here, we show for the first time to our knowledge how thioredoxin activity regulates thiol density expressed on the membrane surface of NK cells, acting as a reducing shield that also protects neighboring $\mathrm{T}$ cells against oxidative stress. With a particular interest in studying NSCLC, we found that NK cells within the tumor core showed higher surface thiol densities than did NK cells found in the tumor periphery and adjacent normal lung tissues. We also found the accumulation of intracellular ROS in NK cells to be higher in the tumor periphery and normal tissues of patients with NSCLC who had a history of tobacco smoking. The Cancer Genome Atlas (TCGA) public database showed that the prognostic value of the NK cell gene signature and IL15 gene expression in the tumor could be influenced by the patient's tobacco smoking history.

\section{Results}

IL-15-primed NK cells mount a superior immune response under oxidative stress. To test whether IL-2 and IL-15 differ in their ability to render NK cells less susceptible to oxidative stress, we analyzed the activity of cytokine-primed NK cells following exposure to $\mathrm{H}_{2} \mathrm{O}_{2}$. At a lower effector-to-target (E/T) ratio, IL-15-primed NK cells showed a greater ability to kill K562 target cells compared with IL-2-primed NK cells (Figure 1A). With exposure to $\mathrm{H}_{2} \mathrm{O}_{2}$ at a dose of $5 \mu \mathrm{M}$ and at an $\mathrm{E} / \mathrm{T}$ ratio of 9:1, we observed that the ability of both IL-2- and IL-15-primed NK cells to kill K562 target cells was not reduced compared with control cells that were not exposed to $\mathrm{H}_{2} \mathrm{O}_{2}$. However, at a lower $\mathrm{E} / \mathrm{T}$ ratio of 3:1, the cytotoxicity of IL-2-primed NK cells was significantly reduced compared with that of IL-15-primed NK cells. Regardless of E/T ratios, the cytotoxicity of both IL-2- and IL-15-primed NK cells was significantly reduced when exposed to a higher $\mathrm{H}_{2} \mathrm{O}_{2}$ dose of $10 \mu \mathrm{M}$. Still, the ability of IL-15-primed NK cells to kill K562 target cells was significantly higher compared with IL-2-primed NK cells (Figure 1B). Similarly, in response to K562 cell stimulation, IL-15-primed NK cells showed significantly higher degranulation and production of IFN- $\gamma$ compared with IL-2-primed NK cells. In the presence of a high $10 \mu \mathrm{M}$ dose of $\mathrm{H}_{2} \mathrm{O}_{2}, \mathrm{IL}-15$-primed NK cells showed significantly higher degranulation and production of IFN- $\gamma$ than did IL-2-primed NK cells (Figure 1, C and D). While intracellular ROS are known to suppress the immune effects of NK cells, we measured the levels of intracellular ROS in cytokine-primed NK cells. These experiments revealed that IL-15-primed NK cells had a reduced accumulation of intracellular ROS compared with IL-2-primed NK cells at the same doses of $\mathrm{H}_{2} \mathrm{O}_{2}$ (Figure 1, $\mathrm{E}$ and F). Neither dose of $\mathrm{H}_{2} \mathrm{O}_{2}$ treatment significantly affected the viability of IL-2- or IL-15-primed NK cells. In terms of cell proliferation, IL-15- and IL-2-primed NK cells were equally susceptible to $\mathrm{H}_{2} \mathrm{O}_{2}$-mediated suppression (data not shown). From these observations, we hypothesized that IL-15-primed cells could intrinsically acquire functions to eradicate ROS from an $\mathrm{H}_{2} \mathrm{O}_{2}$-rich environment and hence maintain their functional activity of killing target cells and producing IFN- $\gamma$.

IL-15 upregulates thioredoxin activity in NK cells by both gene expression and reduced shuttling of mitochondrial TXNIP. To investigate the underlying mechanisms for the increased resistance to oxidative stress by IL-15-primed NK cells, we performed tran- scriptomic analysis of publicly available sequencing data (23). Through gene enrichment analysis, among the top enriched gene ontologies (GOs) (Supplemental Figure 1A; supplemental material available online with this article; https://oi.org/10.1172/ JCI137585DS1) were genes related to oxidoreductase activity (Supplemental Figure 1B). In analyzing genes related to responses to ROS, we identified elevated gene expression of thioredoxins (TXN and TXN2) and reduced expression of their inhibitory counterparts (TXNIP and TXNRD1) (Figure 2A and Supplemental Figure 1, C-F). At the protein level, IL-15-primed cells expressed higher levels of thioredoxin-1 (Figure 2B). However, we performed flow cytometric analysis and found no significant differences in the levels of TXNIP protein expression between IL-2- and IL-15primed NK cells (data not shown). In contrast, we observed differences in subcellular localization of TXNIP. Exposure to $\mathrm{H}_{2} \mathrm{O}_{2}$ induced the release of TXNIP from the nucleus (Figure 2C), and the expression of TXNIP was significantly higher within the nucleus of IL-15-primed NK cells compared with expression in the nucleus of IL-2-primed NK cells (Figure 2D). Furthermore, when exposed to $\mathrm{H}_{2} \mathrm{O}_{2}$, IL-2-primed NK cells, but not IL-15-primed NK cells, resulted in shuttling of TXNIP into the mitochondria, where thioredoxin-2 is expressed (Figure 2E). The beneficial roles of IL-15 in the current context could be explained by the downregulation of CD25 (IL-2 receptor $\alpha$ ) and the upregulation of CD215 (IL15 receptor $\alpha$ ) upon exposure to $\mathrm{H}_{2} \mathrm{O}_{2}$ (Supplemental Figure 1, G and $\mathrm{H}$ ). Expression of CD122 (IL-2 receptor $\beta$ ), the common receptor subunit for both IL-2 and IL-15 signaling, remained unchanged under oxidative stress (Supplemental Figure 1I). These changes in subcellular localization and expression profiles demonstrate how thioredoxin activity is regulated in NK cells to reduce intracellular ROS for improved immune functions of IL-15-primed NK cells.

NK cells have a high surface thiol density that serves as a reducing shield against ROS and sustains cytotoxicity function. In addition to the thioredoxin system that reduces intracellular ROS, cell-surface thiols can act as a protective shield as they are oxidized by external free radicals. We used maleimide, which binds to protein thiols without interfering with their function, and found that IL-15-primed NK cells had significantly higher levels of cellsurface thiols compared with levels on IL-2-primed NK cells (Figure $3 \mathrm{~A}$ ). Following exposure to $\mathrm{H}_{2} \mathrm{O}_{2}$, both IL-2- and IL-15-primed NK cells showed reduced cell-surface thiol density (Figure 3B). Moreover, only NK cells with low thiol density (low maleimide fluorescence intensity) showed an accumulation of intracellular ROS after $\mathrm{H}_{2} \mathrm{O}_{2}$ treatment (Figure 3C). Regardless of stimulation with IL-2 or IL-15, NK cells with high surface thiol density (FACSsorted on the basis of maleimide fluorescence intensity) showed superior killing of $\mathrm{K} 562$ target cells in the presence of $\mathrm{H}_{2} \mathrm{O}_{2}$ (Figure 3D). In analyzing the regulation of thioredoxin and the NK cell phenotype, we found that NK cells with high surface thiol density also expressed higher levels of CD56, CD16, and thioredoxin. These NK cells also displayed increased mTOR activation as measured by the phosphorylation of S6 kinase (S6K) (Figure 3E). In terms of subcellular location, TXNIP was enriched in the cytoplasm in NK cells with lower surface densities of thiol (Figure $3 \mathrm{~F}$ ). These results indicated that the density of surface thiols on NK cells is associated with favorable antitumor functions and resistance against ROS-mediated immune suppression. 
A

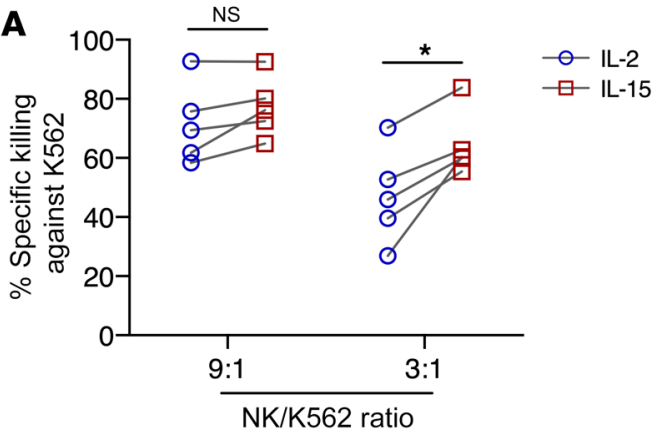

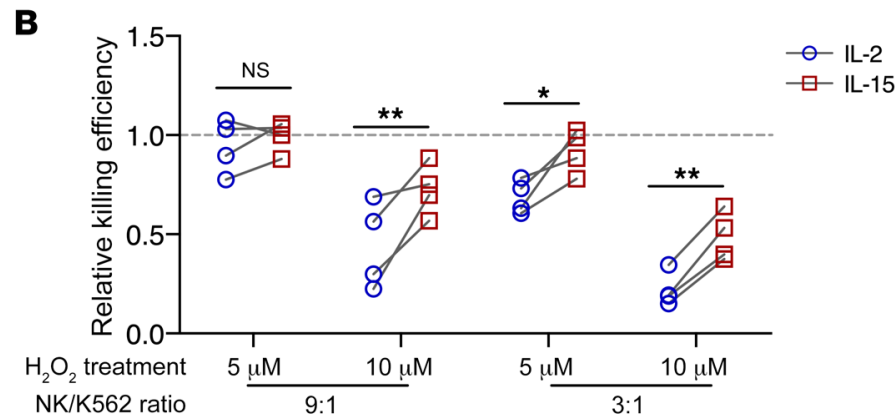

C

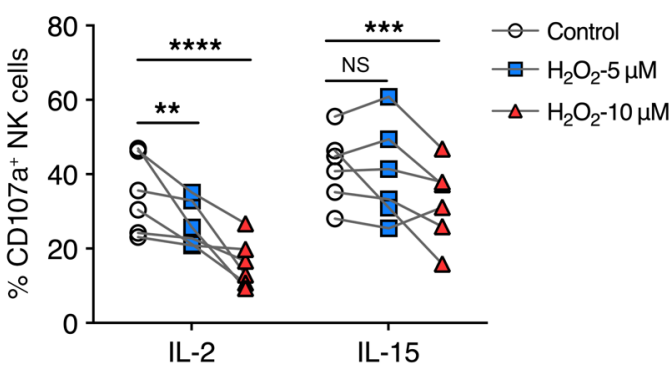

D
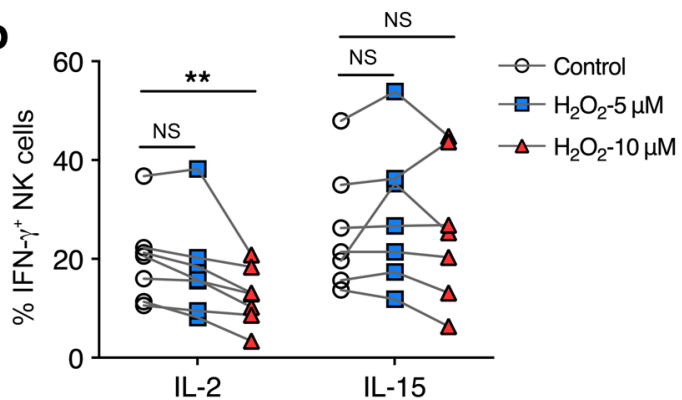

$\mathbf{E}$

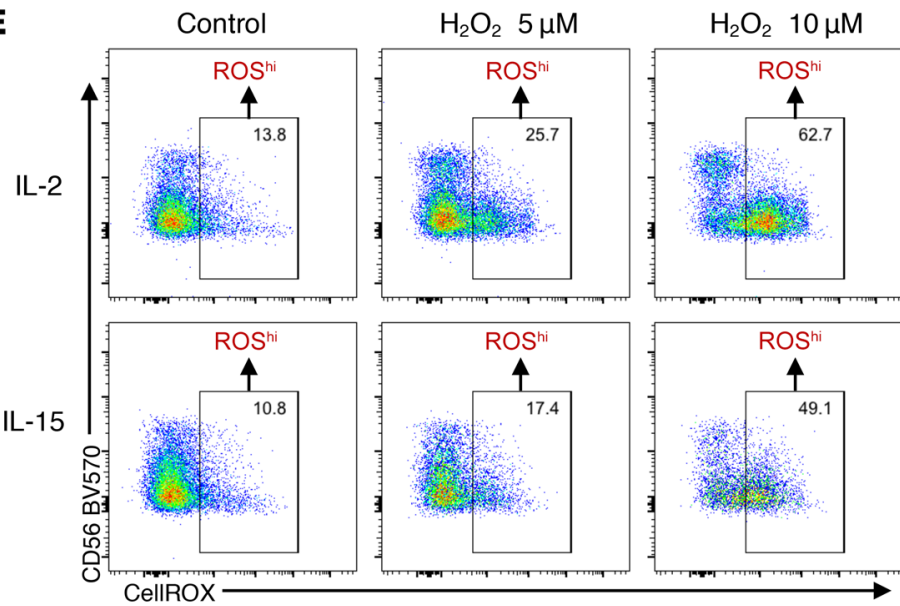

$\mathbf{F}$

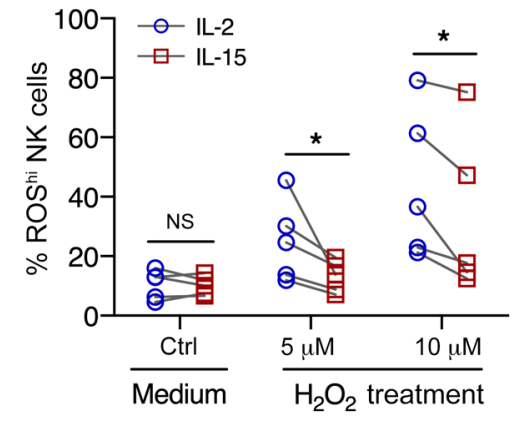

Figure 1. IL-15-primed NK cells mount a superior immune response under oxidative stress. (A) Percentage of specific killing of K562 target cells by NK cells primed with either IL-2 or IL-15 and cocultured at 2 different E/T ratios $(n=5)$. (B) Relative killing efficiency at two $E / T$ ratios of NK cells primed with either IL-2 or IL-15, normalized to the control without $\mathrm{H}_{2} \mathrm{O}_{2}$ treatment $(n=4)$. (C) Percentage of CD107a+ NK cells primed with either IL-2 or IL-15, in the absence or presence of $\mathrm{H}_{2} \mathrm{O}_{2}$ treatment. (D) Percentage of IFN- $\gamma^{+} \mathrm{NK}$ cells primed with either IL-2 or IL-15, in the absence or presence of $\mathrm{H}_{2} \mathrm{O}_{2}$ treatment. (E) Representative FACS plots showing the gating strategy for NK cells with high intracellular ROS $(n=5)$. (F) Percentage of IL-15- and IL-2-primed NK cells with high intracellular ROS after $\mathrm{H}_{2} \mathrm{O}_{2}$ treatment $(n=5) .{ }^{*} P<0.05$, ${ }^{* *} P<0.01$, ${ }^{* *} P<0.001$, and ${ }^{* * * *} P<0.0001$, by mixed-model analysis with HolmŠidák's multiple-comparisons test (A-F). All individual data points are connected for matching replicates.

Inhibition of thioredoxin-1 reduces NK cell-surface thiol density and reverses IL-15-mediated resistance against oxidative stress. Since thioredoxins and surface thiols are expressed at higher levels in IL-15-primed NK cells, we sought to investigate whether the thioredoxin system plays a role in the regulation of surface thiols and to validate that thioredoxin- 1 has an important role in conferring resistance to ROS-mediated immune suppression. In the presence of the selective thioredoxin-1 inhibitor PX-12, surface thiols were significantly reduced on both IL-2- and IL-15-primed NK cells (Figure 4A). Additionally, under PX-12 inhibition, surface thiols on both IL-2- or IL-15-primed NK cells were reduced to a lesser extent in the presence of $\mathrm{H}_{2} \mathrm{O}_{2}$ (Supplemental Figure 2A). Upon
PX-12 treatment, we detected similar levels of accumulated ROS in both IL-2- and IL-15-primed NK cells (Figure 4B). In addition, the presence of PX-12 abrogated the ability of IL-15-primed NK cells to kill K562 cells upon exposure to $\mathrm{H}_{2} \mathrm{O}_{2}$ (Supplemental Figure 2B). Using a physiological model of oxidative stress, we cultured NK cells with autologous activated neutrophils. In these experiments, PX-12 treatment resulted in higher intracellular ROS levels in the IL-15-treated cells (Figure 4C). Moreover, ROS produced by the activated neutrophils suppressed the proliferation of NK cells, and treatment with PX-12 reduced the proliferation of IL-15-primed NK cells to the same levels of untreated IL-2-primed NK cells (Figure 4D). 
A

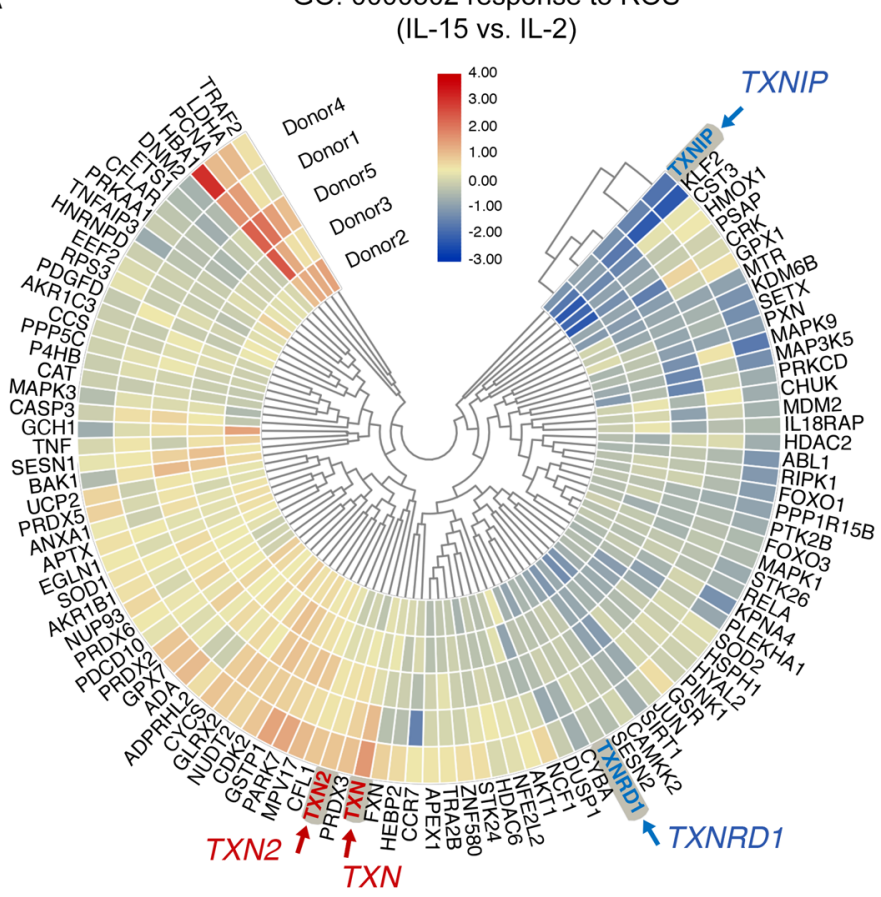

B
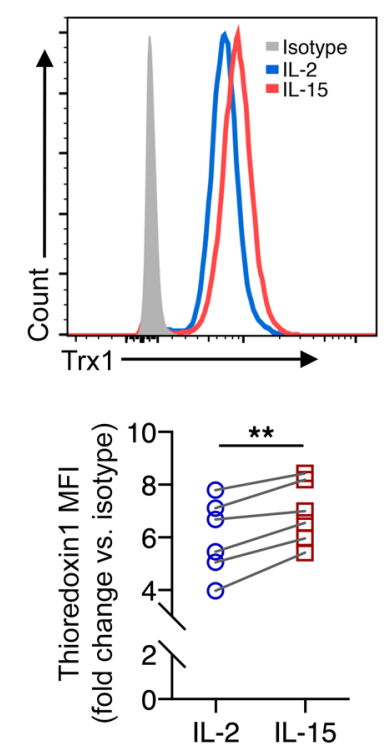

D

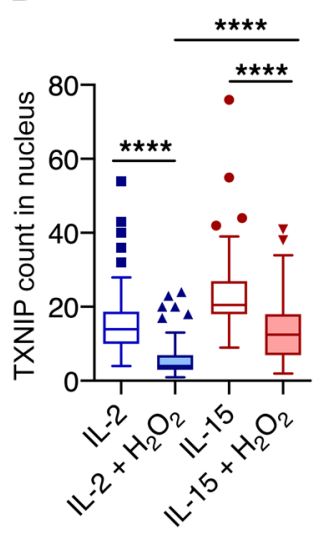

E

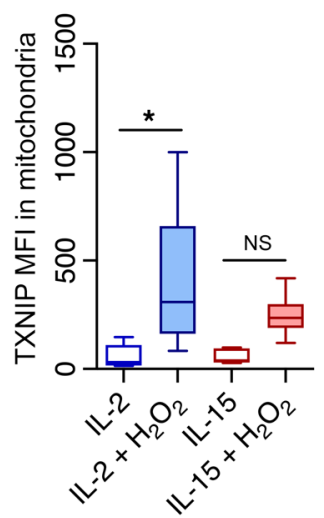

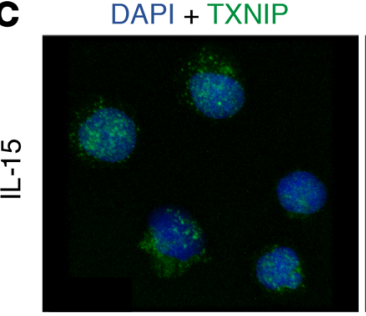

DAPI + MitoTracker
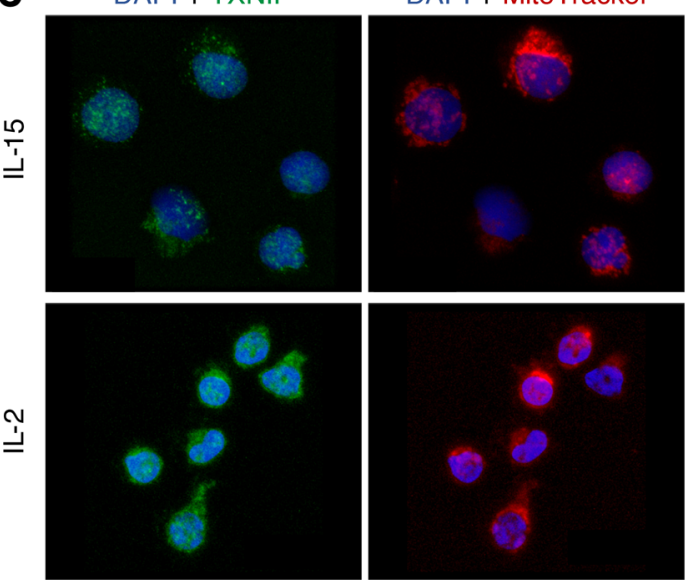
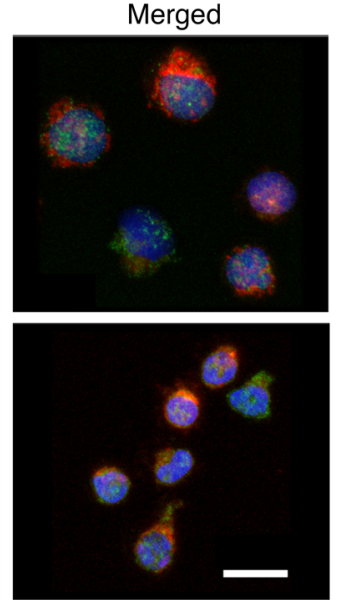

Figure 2. IL-15 upregulates thioredoxin activity in NK cells and reduces shuttling of TXNIP into mitochondria. (A) Heatmap of differential gene expression in GO (GO:000302) based on comparisons of IL-15- and IL-2-primed NK cells. (B) Representative histogram and relative MFI of thioredoxin (Trx1) in NK cells, normalized to the isotype control. (C) Maximum-intensity projections of confocal images ( $\times 63$ objective) for NK cells treated with $10 \mu \mathrm{M} \mathrm{H} \mathrm{O}_{2}$ and primed with either IL-15 or IL-2. Blue (DAPI) shows staining of nuclei, green shows staining for TXNIP, and red shows staining of mitochondria. Scale bar: $10 \mu \mathrm{m}$. (D) Image quantification of TXNIP counts (green objects) per cell nucleus in NK cells with or without $10 \mathrm{mM} \mathrm{H}_{2} \mathrm{O}_{2}$ treatment. (E) Image quantification of TXNIP MFI (green fluorescence intensity) overlapping with mitochondria (red objects) in NK cells with or without $10 \mu \mathrm{M} \mathrm{H}_{2} \mathrm{O}_{2}$ treatment. ${ }^{*} P<0.05$, ${ }^{* *} P<0.01$, and ${ }^{* * *} P<0.0001$, by Wilcoxon signed-rank test $(\mathbf{B})$ and Kruskal-Wallis test ( $\mathbf{D}$ and $\mathbf{E}$ ). Data were pooled from 3 biological replicates and are represented as Tukey's box plots.

To determine whether NK cells could be affected by ROS produced by tumor cells, we used a lung adenocarcinoma 3D sphere model (Supplemental Figure 2C). NK cells that infiltrated and localized inside the sphere were found to accumulate more intracellular ROS (Supplemental Figure 2D). Inhibition of thioredoxin-1 resulted in a significant reduction of infiltration by IL-15primed NK cells (Figure 4, E and F). Furthermore, purified NK cells with high surface thiol density showed a superior ability to infiltrate lung tumor spheres (Figure 4G). The removal of surface thiols by $N$-ethylmaleimide in IL-15-primed NK cells also resulted in reduced infiltration into tumor spheres (Supplemental Figure $2, \mathrm{E}$ and $2 \mathrm{~F}$ ). In contrast, the addition of exogenous thioredoxin-1 instead promoted the infiltration of IL-2-primed NK cells into the tumor spheres (Supplemental Figure 2G). Taken together, we found a causal relationship between NK cell thioredoxin-1 expression and surface thiol density. In 2 separate physiological models of ROS, IL-15-primed NK cells showed an increased ability to resist ROS-meditated immune suppression. 
A

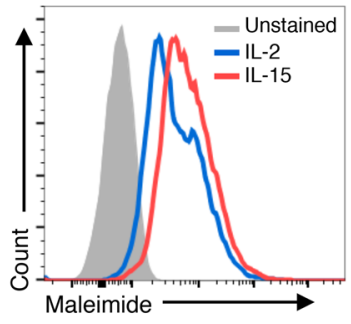

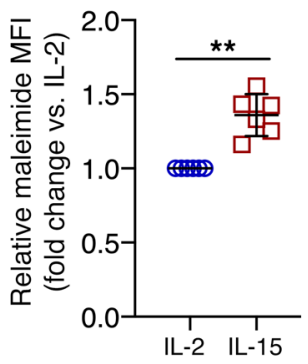

B

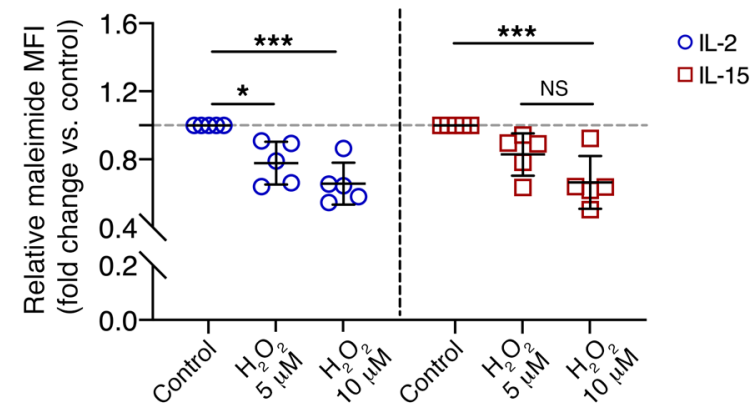

C

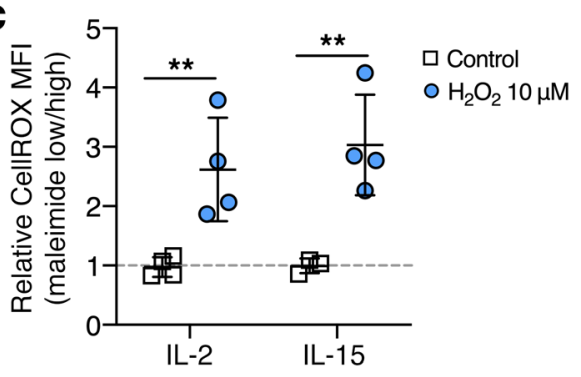

$\mathbf{E}$

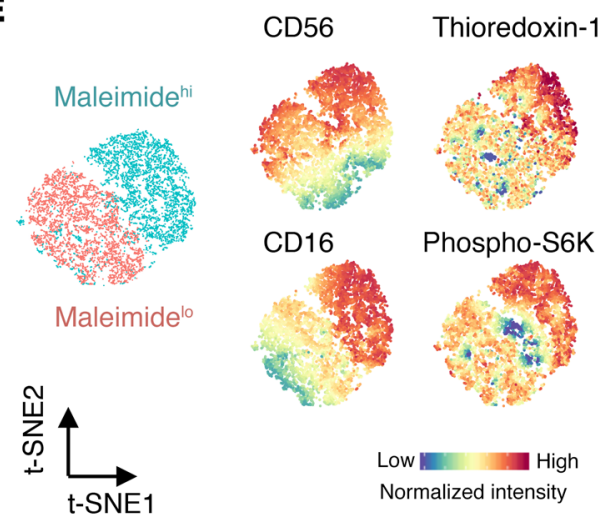

D

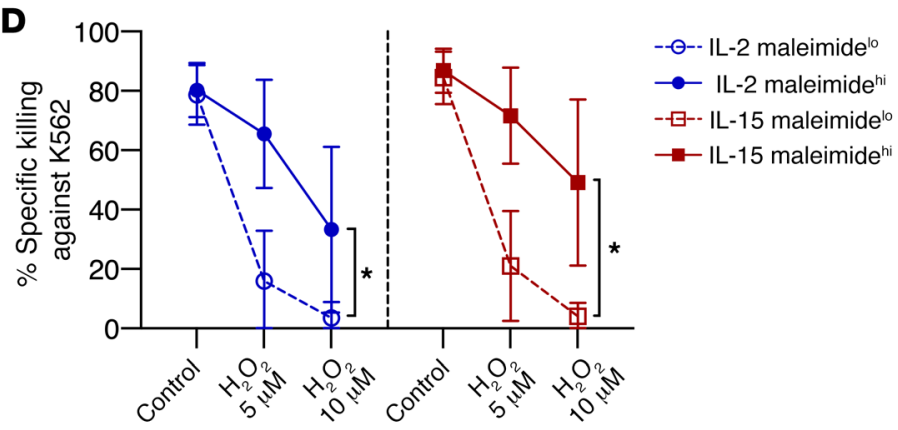

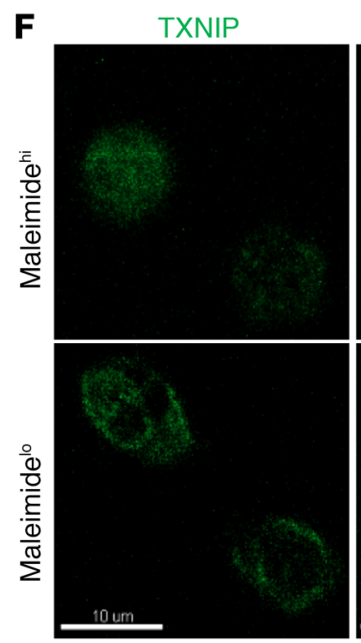
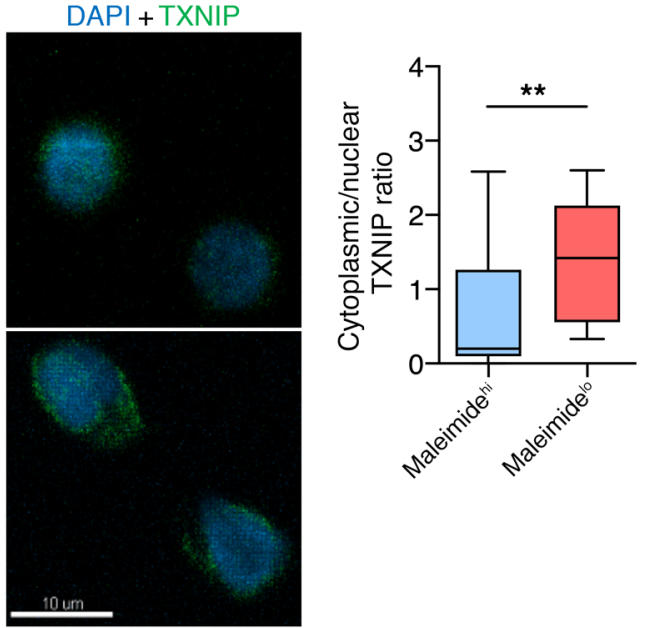

Figure 3. NK cells express surface thiol groups to overcome oxidative stress and sustained cytotoxicity function. (A) Representative histogram and relative MFI of maleimide staining comparing IL-2- and IL-15-primed NK cell cultures $(n=6)$. (B) Relative MFI of maleimide staining comparing cells with 1 hour of $\mathrm{H}_{2} \mathrm{O}_{2}$ treatment in both IL-2- and IL-15-primed NK cell cultures $(n=5)$. (C) Relative MFI of intracellular ROS based on CellROX staining normalized to maleimide ${ }^{10} \mathrm{NK}$ cells $(n=4)$. (D) Percentage of specific killing of K562 target cells at a 5:1 E/T ratio, with effector NK cells presorted on the basis of maleimide staining $(n=4)$. (E) t-SNE analysis of NK cells based on surface thiol density (maleimide ${ }^{\text {hi }}$ vs. maleimide ${ }^{l 0}$ ) and its phenotypes acquired by flow cytometry. (F) Representative $\times 63$ maximum-intensity projections of TXNIP localization within NK cells sorted by surface density and relative quantification of TXNIP in cytoplasm versus the nucleus. Green shows TXNIP staining and blue shows DAPI staining of the nucleus. Scale bars: $10 \mu \mathrm{m}$. Data were pooled from 3 biological replicates and are represented as Tukey's box plots. All Individual data points are presented as the mean $\pm S D$. ${ }^{*} P<0.05$, ${ }^{* *} P<$ 0.01 , and ${ }^{* * *} P<0.001$, by Mann-Whitney $U$ test $(\mathbf{A}$ and $\mathbf{F})$, repeated-measures 2-way ANOVA with Holm-Šidák's multiple-comparisons test (B and $\left.\mathbf{D}\right)$, and ordinary 2-way ANOVA with Tukey's test for multiple-comparisons (C).

mTOR activation suppresses the expression of TXNIP to sustain thioredoxin activity and cell surface thiol density. We previously identified mTOR activation as an important difference for the discrimination of functional outcomes between IL-15- and IL-2primed NK cells (23). To determine whether mTOR activation is linked to the expression of thioredoxin, thioredoxin-interacting protein (TXNIP), and cell-surface thiol density, the selective mTOR inhibitor Torin-1 was added to cytokine-primed NK cells. Indeed, the presence of Torin-1 resulted in upregulation of
TXNIP in IL-15-primed NK cells (Figure 5A). Upon Torin-1 treatment, we observed a reduction in thioredoxin activity (Figure $5 B)$. Notably, the difference in thioredoxin expression between IL-2- and IL-15-primed NK cells diminished upon treatment with Torin-1 (Figure 5C). Similarly, we also found no significant differences in cell-surface thiol density or accumulation of intracellular ROS between IL-2- and IL-15-primed NK cells upon treatment with Torin-1 (Figure 5, D and E). Addition of the mTOR agonist MHY1485 also increased cell-surface thiol den- 
A
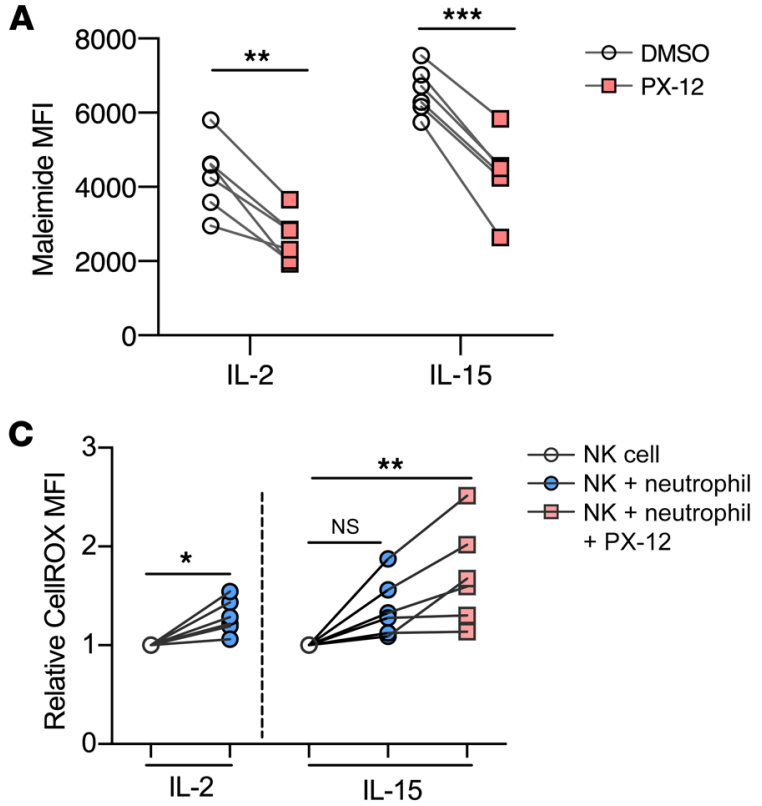

B

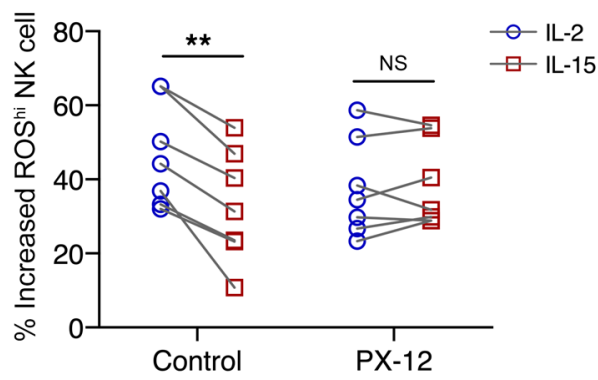

D

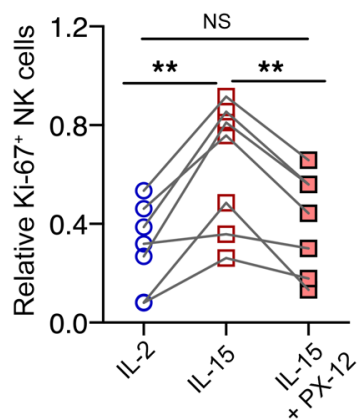

$\mathbf{E}$

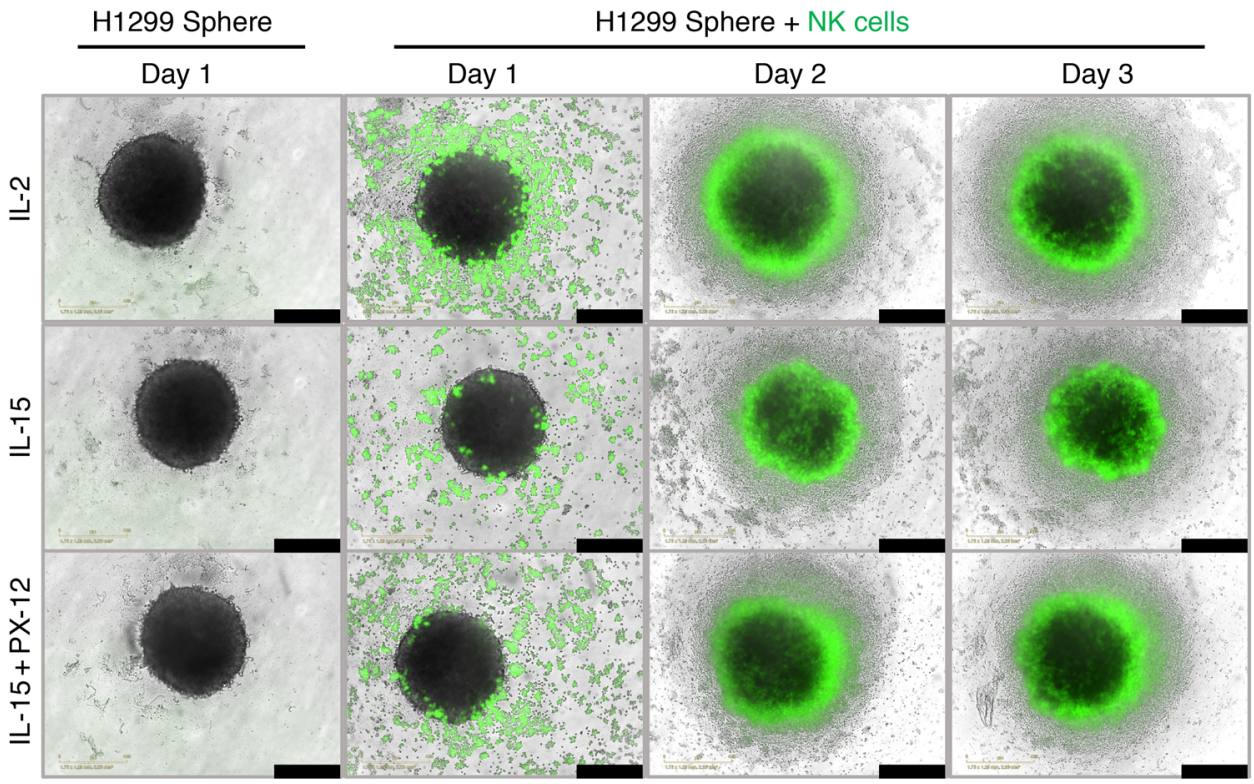

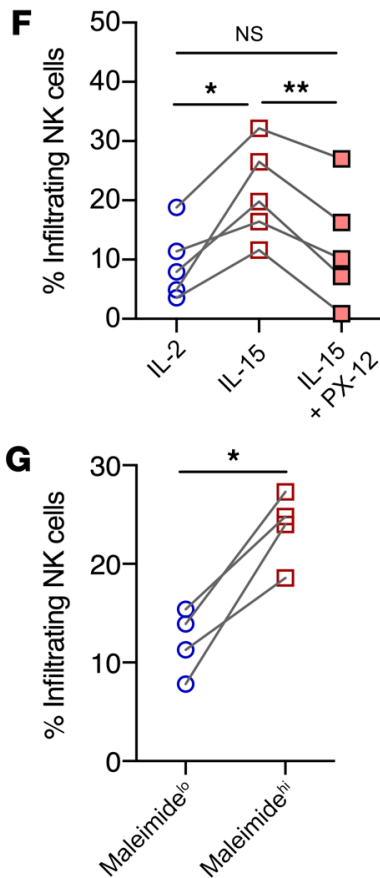

Figure 4. Inhibition of thioredoxin-1 reduces NK cell surface thiol groups and reverses IL-15-mediated resistance to oxidative stress. (A) MFI of maleimide staining on NK cells treated with PX-12 within IL-2- and IL-15-primed NK cell cultures $(n=6)$. (B) Percentage of increased ROS hi NK cells after $10 \mu \mathrm{M} \mathrm{H} \mathrm{O}_{2}$ treatment compared with untreated NK cells in the presence or absence of PX-12 in IL-2 and IL-15 cultures $(n=6)$. (C) Relative increase in CellROX MFI in NK cell cocultures with or without PX-12 treatment, normalized to the control group without neutrophils $(n=6)$. (D) Relative fold-change of Ki-67 expression in NK cell cocultures with or without PX-12 treatment $(n=7)$. (E) Bright-field images of H1299 tumor spheres with green fluorescence-labeled NK cells. Images were acquired under a $\times 10$ objective at 3 different time points. Scale bars: $400 \mathrm{~mm}$. Vertical labels describe the pretreatment conditions of NK cells before coculture. (F) Percentage of infiltrating NK cells in tumor spheres with different NK cell pretreatments $(n=5)$. (C) Percentage of infiltrating NK cells in tumor spheres in FACS-sorted NK cells, based on maleimide staining $(n=4)$. All individual data points are connected for matching replicates. ${ }^{*} P<0.05$, ${ }^{* *} P<0.01$, and ${ }^{* *} P<0.001$, by repeated-measures 2-way ANOVA with Holm-Šidák's multiple-comparisons test (A and B), Wilcoxon signed-rank test (for significance within IL-2 cultures) and Friedman's test (for significance within IL-15 cultures) (C), Friedman's test (D and F), and paired $t$ test (G).

sity and reduced the accumulation of intracellular ROS in NK cells (data not shown). Treatment with Torin-1 also resulted in the shuttling of TXNIP out of the nucleus in IL-15-primed NK cells (Figure 5, F-H). These findings demonstrated that mTOR is a key regulator of the thioredoxin system and, hence, of the IL-15-mediated resistance against oxidative stress.
NK cell infiltration is influenced by the accumulation of oxidative stress in NSCLC tumors. From a cohort of patients with NSCLC (see Supplemental Table 1 for the patients' clinical characteristics), we detected a higher frequency of CD56 lymphocytes within tissue sections obtained from the tumor periphery as compared with the tumor core (Figure 6A). In a more detailed analysis in which sam- 
A

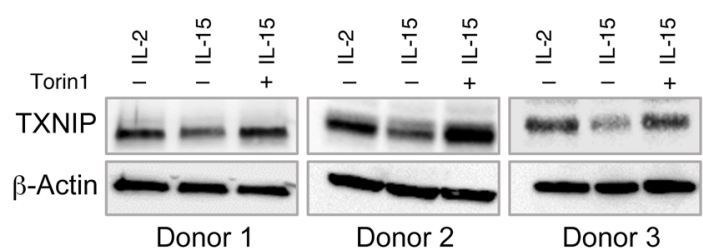

B

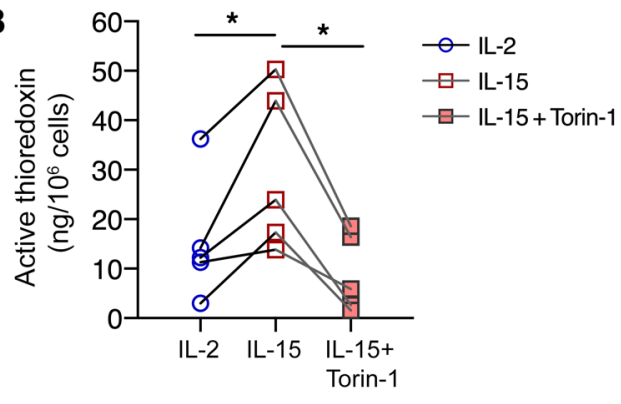

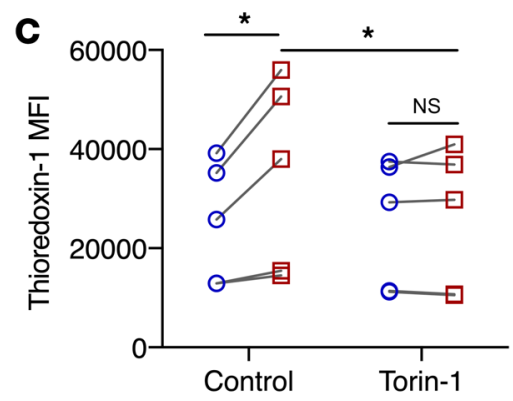

D

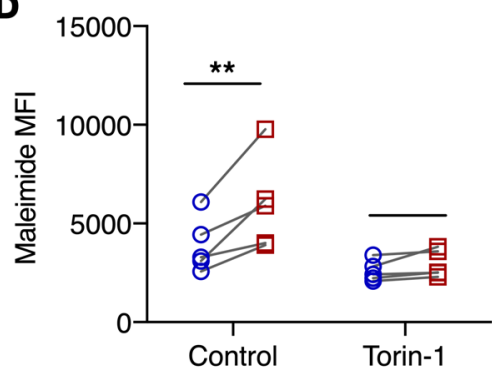

E

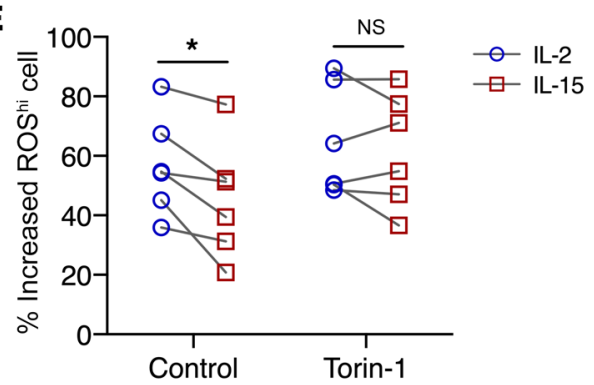

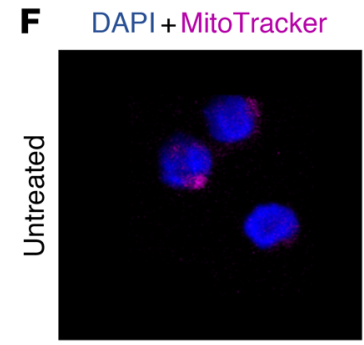
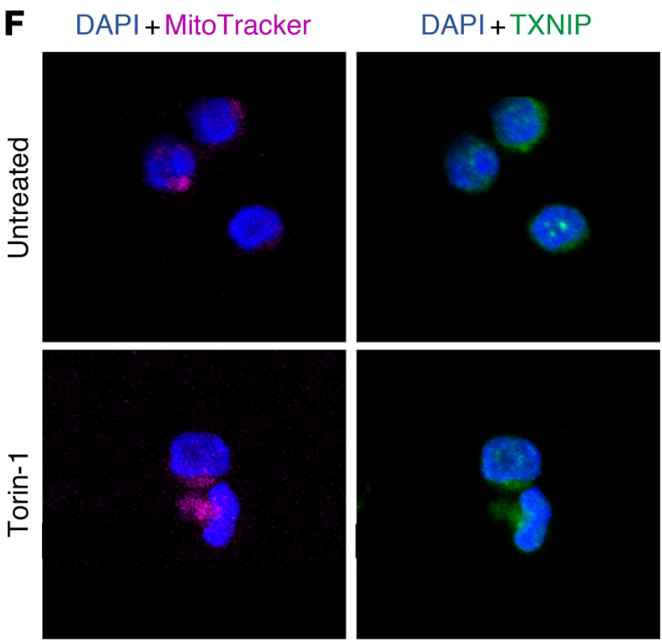
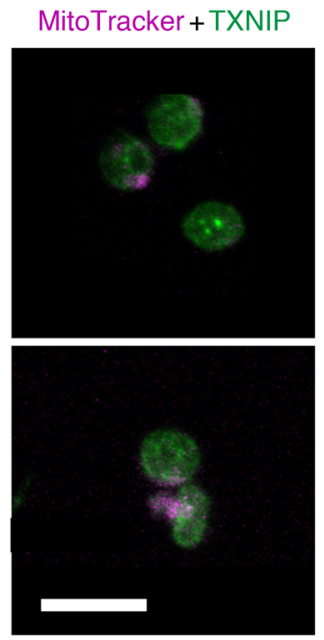
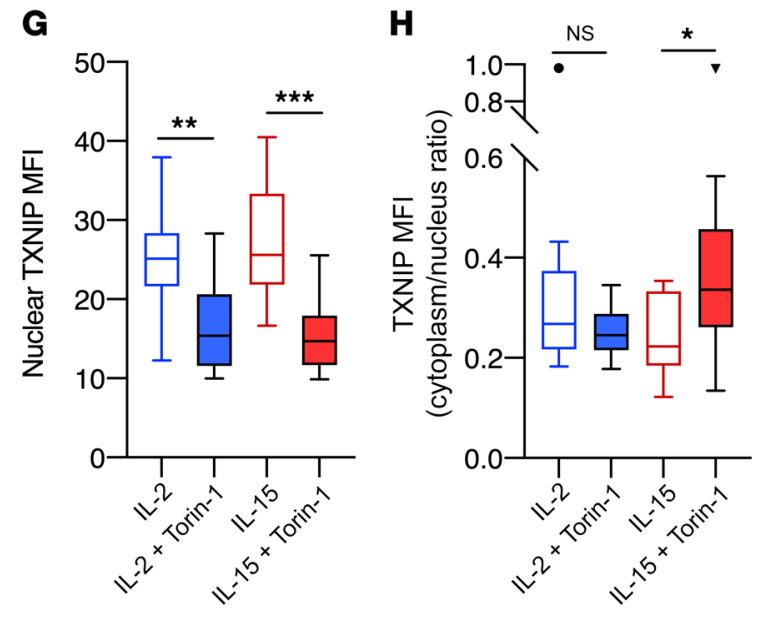

Figure 5. mTOR suppresses TXNIP expression to sustain thioredoxin activity and surface thiol density. (A) Western blots of TXNIP protein expression in NK cells treated with Torin-1 $(n=3)$. (B) Concentration of active thioredoxin protein in NK cells primed with either IL-2 or IL-15 or with IL-15 plus Torin-1 treatment $(n=5)$. (C) MFI of thioredoxin-1 expressed in IL-12- or IL-15-primed NK cells with and without Torin-1 treatment $(n=5)$. (D) MFI of maleimide staining of IL-12- or IL-15-primed NK cells with and without Torin-1 treatment $(n=5)$. (E) Relative increased percentage of ROS hi NK cells primed with either IL-2 or IL-15 and treated with Torin-1 after exposure to $10 \mu \mathrm{M} \mathrm{H}_{2} \mathrm{O}_{2}$, compared with NK cells without $\mathrm{H}_{2} \mathrm{O}_{2}$ exposure $(n=6)$. (F) Representative maximum-intensity projections of confocal images ( $\times 63$ objective) of NK cells treated with $1 \mathrm{mM}$ Torin-1. Blue represents DAPI staining of the nucleus, green represents TXNIP staining, and magenta represents mitochondria staining. Scale bar: $10 \mu \mathrm{m}$. (C) MFI of TXNIP (green fluorescence intensity) within the nuclei of NK cells under $\mathrm{H}_{2} \mathrm{O}_{2}$ oxidative stress. (H) Image quantification of the cytoplasm/nucleus ratio of TXNIP MFI of NK cells under $\mathrm{H}_{2} \mathrm{O}_{2}$ oxidative stress. All individual data points are connected for matching replicates. Data were pooled from 3 biological replicates and are represented as Tukey's box plots. ${ }^{*} P<0.05$, ${ }^{* *} P<0.01$, and ${ }^{* * *} P<$ 0.001, by Friedman's test (B), repeated-measures 2-way ANOVA with Holm-Šidák's multiple-comparisons test (C-E), and Kruskal-Wallis test (G and $\mathbf{H})$.

ples were segregated into the different tissue locations - tumor core, tumor periphery, and adjacent normal lung tissue (Supplemental Figure 3A) - we observed significantly higher frequencies of NK cells in the peripheral tumor region and normal adjacent tissue compared with the tumor core (Figure 6B). NK cells within the tumor core showed significantly higher intracellular ROS levels compared with levels in NK cells residing in the tumor periphery and adjacent normal tissues (Figure 6C). In addition, we observed a significant negative correlation between the levels of intracellular ROS and the frequency of tissue-infiltrating NK cells (Figure
6D). When we segregated the data obtained from different tissue regions, we found a significant and strong negative correlation in normal tissue and the tumor periphery but not in the tumor core (Figure 6, E-G). Given that these tissues in general displayed high positivity for 8-OHdG expression (Supplemental Figure 3B), oxidative stress had significant effects on NK cell infiltration and the accumulation of intracellular ROS within NSCLC tumors.

The NK cell signature and IL15 gene expression predict a better prognosis in patients with NSCLC who have a smoking history. Our analysis of TCGA data revealed that expression of the IL15 gene 
A

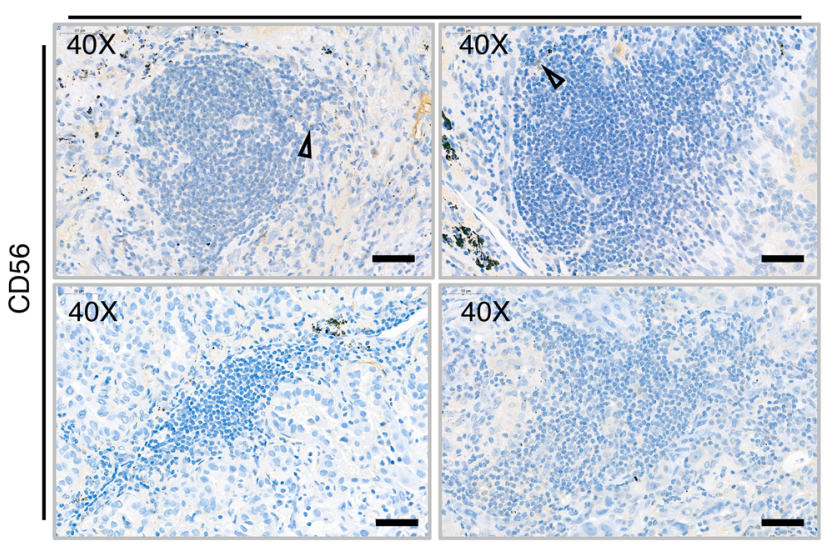

Tumor periphery
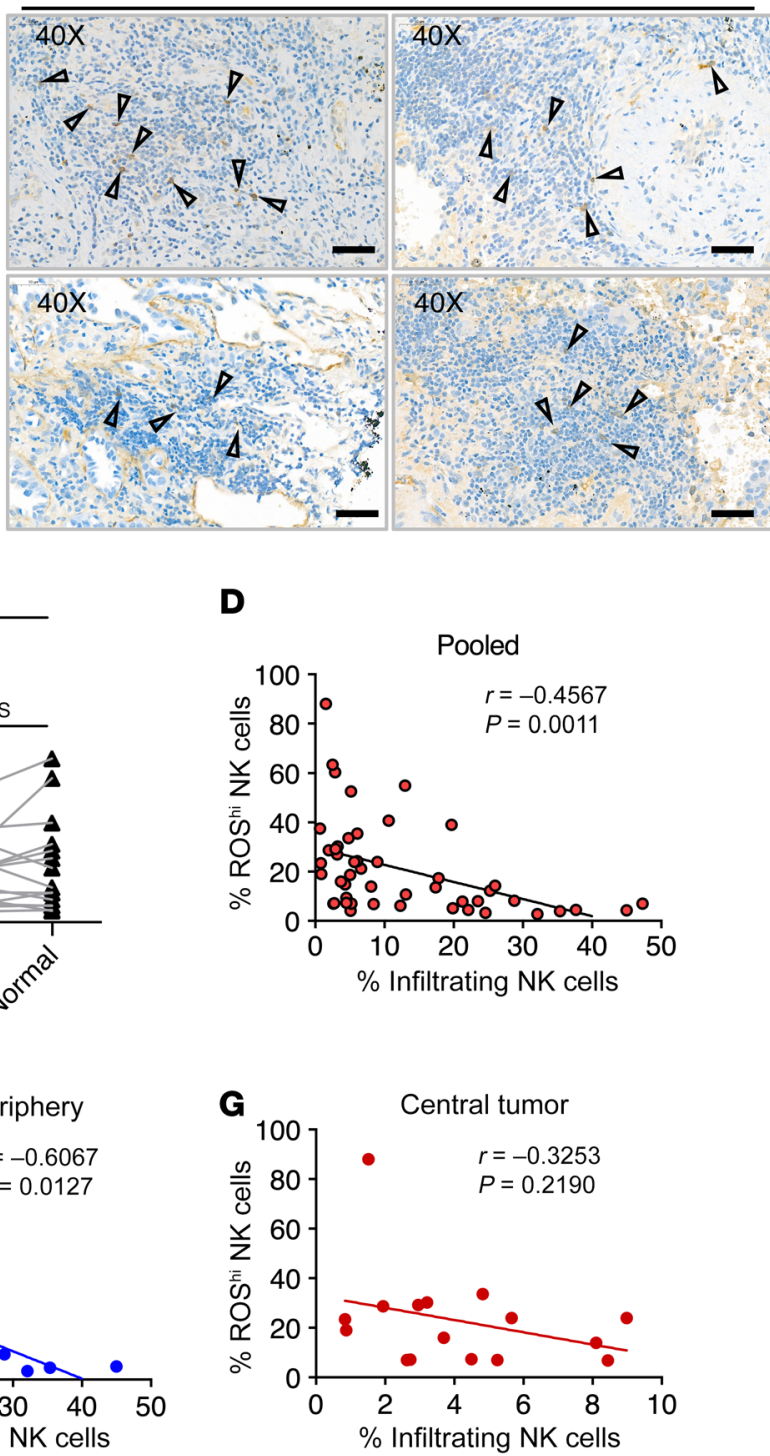

Figure 6. NK cell infiltration is influenced by the accumulation of oxidative stress in NSCLC tumors. (A) Representative images of immunohistochemical staining for CD56 within immune infiltrates found in NSCLC tumor core and tumor periphery. Triangles indicate CD56+ lymphocytes. Scale bars: 50 $\mu \mathrm{m}$ (original magnification, $\times 40$ ). $n=4$. (B) Percentage of infiltrating NK cells in the different patient tissues collected $(n=16)$. (C) Percentage of ROS ${ }^{\text {hi }}$ NK cells in the different patient tissues collected $(n=16)$. (D-G) Correlation of ROS ${ }^{\text {hi }}$ NK cells with the percentage of infiltrating NK cells isolated in (D) pooled tissue samples $(n=48)$, (E) adjacent normal lung tissue $(n=16)$, (F) tumor periphery $(n=16)$, and (C) central tumor $(n=16)$. (B and C) All matching data points for autologous samples are connected with lines. ${ }^{* *} P<0.01,{ }^{* * *} P<0.001,{ }^{* * *} P<0.0001$, by Friedman's test. (D-C) Spearman's rank correlation coefficient test was used to determine significant correlations.

in bulk tumor sequencing was positively correlated with several NK cell gene signature scores within the NSCLC cohort (Supplemental Figure 3C). To confirm our results of the association of NK cell infiltration and oxidative stress, we performed analysis of TCGA data. Since tobacco smoking is known to induce tissue ROS production, we segregated data from the TCGA-Lung Adenocarcinoma (TCGA-LUAD) data set into smoker and nonsmoker cohorts (Supplemental Figure 3D). When we analyzed a set of oxidative stress-related genes that were found to correlate with worse prognosis in lung cancer (24), we found that smokers had a higher score for an oxidative stress gene signature (Supplemental Figure 3E). Although neither the NK cell signature nor IL15 gene expression alone correlated with a better prognosis in the NSCLC cohort (data not shown), the prognostic values of the NK cell signature and IL15 gene expression were influenced by the patients' smoking history. Remarkably, an NK cell gene signature predicted overall survival (OS) and a progression-free interval (PFI) only in the smoker cohort (Figure 7, A and B), but no significant predictive correlation was found in the nonsmoker group (Supplemental Figure 3, F and G). Similarly, IL15 gene expression was only predictive for PFI in the smoker cohort (Figure 7C and Supplemental Figure $3 \mathrm{H})$. From our NSCLC patient cohort in which FACS phenotyping was performed, we observed a significantly higher level of intracellular ROS in NK cells residing in the tumor periphery and adja- 
A

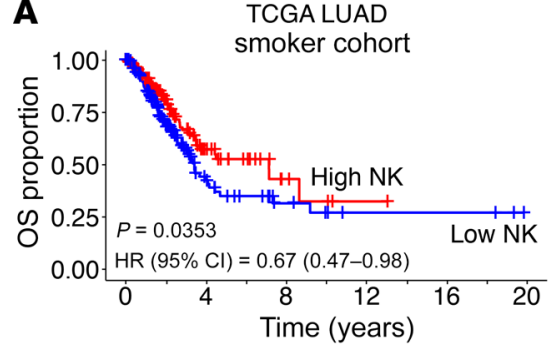

\begin{tabular}{cccccc} 
High NK.158 & 28 & 6 & 1 & 0 & 0 \\
Low NK.158 & 25 & 8 & 3 & 3 & 0 \\
\hline 0 & 4 & 8 & 12 & 16 & 20 \\
& & \multicolumn{1}{c}{$\begin{array}{c}1 \\
\text { Time (years) }\end{array}$} & &
\end{tabular}

\section{D}

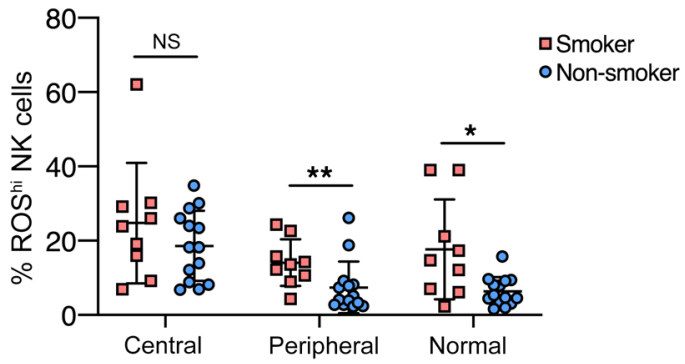

B

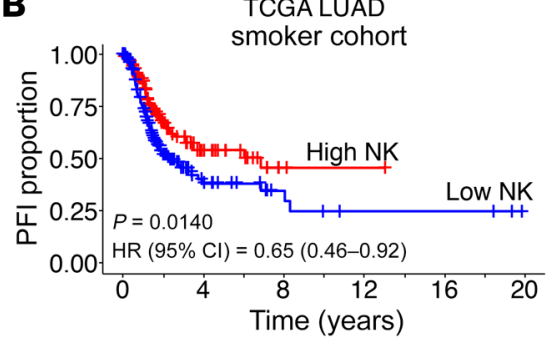

C

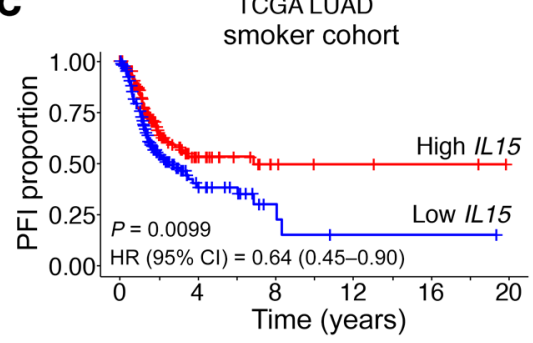

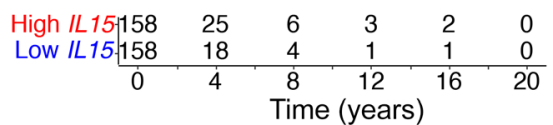
Time (years) (1)

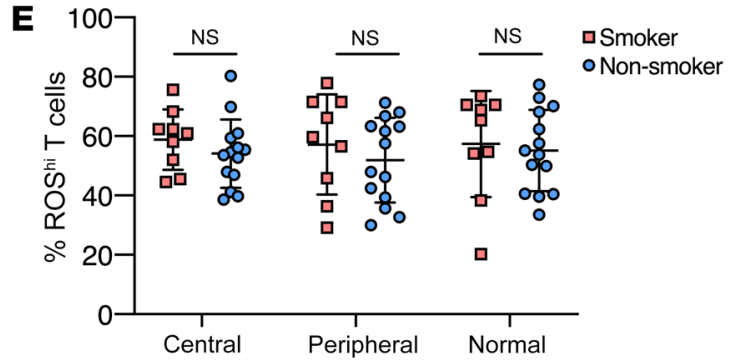

Figure 7. NK cell signature and IL15 gene expression predict a better prognosis in patients with NSCLC who have a smoking history. (A) OS of TCCANSCLC smoker cohort based on the median NK gene signature score. (B) PFI of TCGA-NSCLC smoker cohort based on the median NK gene signature score. (C) PFI of TCCA-NSCLC smoker cohort based on median IL15 gene expression. (A-C) A log-rank test was used to determine significance in differences in survival distribution $(n=316)$. (D) Percentage of ROS ${ }^{\text {hi }}$ NK cells in different patient tissues classified by the patients' smoking history. (E) Percentage of ROS $^{\text {hi }}$ T cells in different patient tissues classified by the patients' smoking history. (D and E) Individual data points indicate the mean \pm SD ( $n=9$ smokers; $n=14$ nonsmokers). ${ }^{*} P<0.05$ and ${ }^{* *} P<0.01$, by ordinary 2-way ANOVA with Holm-Šidák's multiple-comparisons test.

cent normal lung tissue of smokers (Figure 7D). Notably, this trend in ROS accumulation was not observed in the context of tissueinfiltrating T cells (Figure 7E).

High surface thiol density on tumor-infiltrating NK cells improves immune infiltration into tumors. To validate that high surface thiol density on NK cells would improve infiltration into tumors, we analyzed the expression of surface thiols on NK cells residing in the tumor core, periphery, and adjacent normal tissues surgically resected from patients with NSCLC. NK cells within the tumor core expressed the highest surface thiol densities as compared with those in the tumor periphery and normal tissue (Figure 8, $\mathrm{A}$ and $\mathrm{B})$. In a previously published data set for NSCLC tumors and peripheral blood, we found that larger proportions of $\mathrm{CD} 45^{+}$ tumor-infiltrating lymphocytes (TILs) including $\mathrm{CD}^{+} \mathrm{T}$ cells and NK cells had a higher enrichment for pathways related to oxidoreductase and thioredoxin disulfide reductase activities compared with cells from peripheral blood (Figure 8C). Focusing on the NK cell population, the normalized enrichment scores (NESs) for these $2 \mathrm{GO}$ pathways were higher in tumor-infiltrating NK cells than in peripheral blood NK cells (Supplemental Figure 4, A and B). We pooled NK cell data from both blood and tumor samples and found that cells enriched for either of these $2 \mathrm{GO}$ pathways also had higher cytotoxicity gene set scores (Supplemental Figure 4, C and D). Although high thioredoxin activity was associated with enhanced cytotoxicity functions, purified NK cells with high and low surface thiol density were tested for their ability to protect neighboring TILs from ROS-mediated immune suppression in a bystander fashion. Using autologous patient-derived tumor spheres, we observed improved autologous TIL infiltration upon addition of NK cells sorted for high thiol density compared with addition of NK cells sorted for low thiol density (Figure 8, D and E). Through the expansion of TILs from patients with NSCLC and sarcoma (see Supplemental Table 2 for the patients' clinical characteristics), IL-15 expanded a higher frequency of TIL-NK cells with higher surface thiol density (Figure 8F). In these TIL cultures, we observed a significant increase in the frequency of NK cells and dividing NK cells only in IL-2 cultures treated with the ROS scavenger catalase (Supplemental Figure 4, E and F).

\section{Discussion}

ROS can be produced by several cell types within the tumor microenvironment and accumulate along with tumor progression. It is well established that NK cells are susceptible to ROS-mediated immune suppression. Often, myeloid cells within the TME produce high levels of ROS to inhibit the antitumor functions of NK cells, which has implications for tumor progression and the formation of distant metastasis (1-3). In addition, clinically approved antibodies such as rituximab can trigger ROS production by myeloid cells, suggesting oxidative stress as a means of resistance against NK cell-dependent therapies (25). Here, we compared the ability of the 2 types of cytokines, IL- 2 and IL-15, to modulate the response of NK cells to ROS and found that IL-15 rendered NK cells less susceptible to oxidative stress through the increased thioredoxin system within the TME.

The expression of thioredoxin is commonly associated with TCR signaling and immune activation (10, 26-28). Early stud- 
A

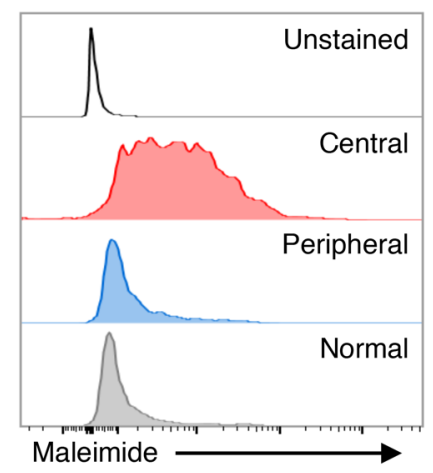

B

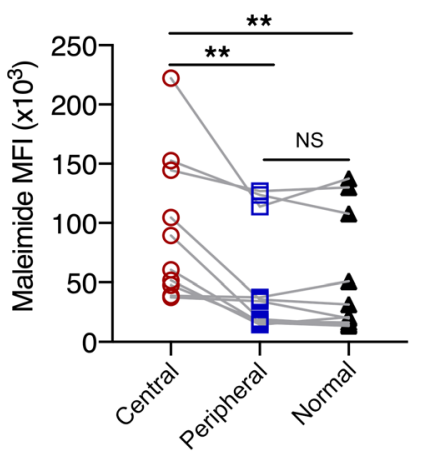

C
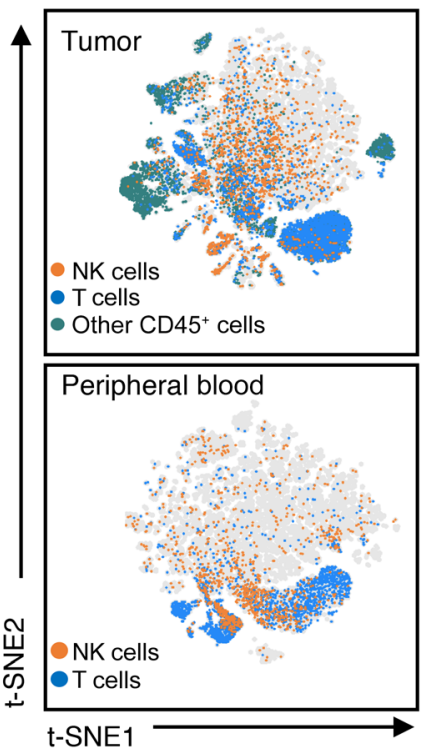

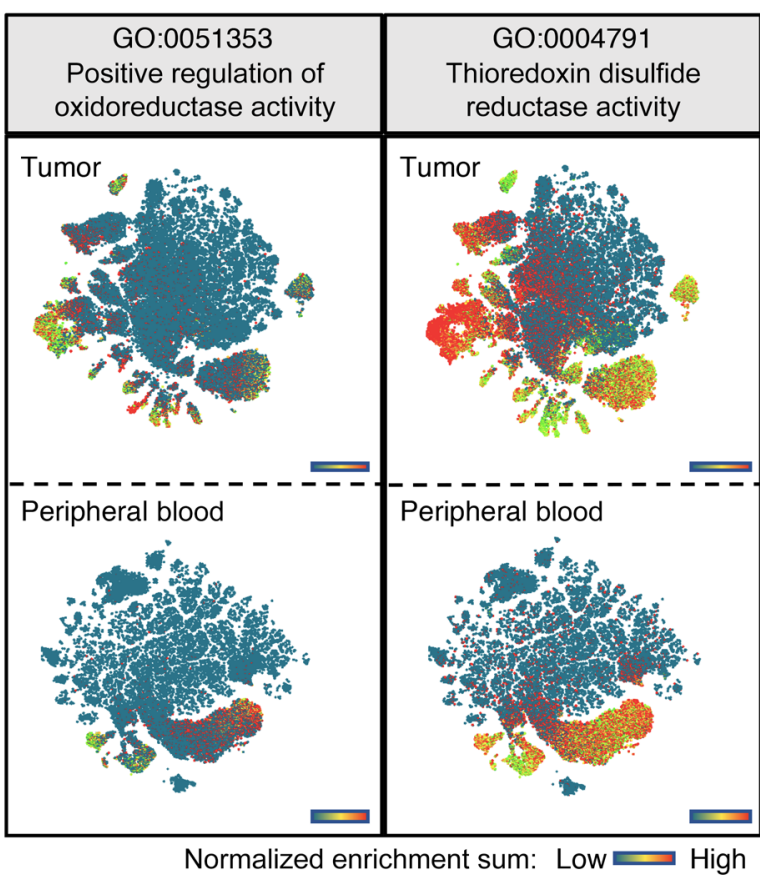

D

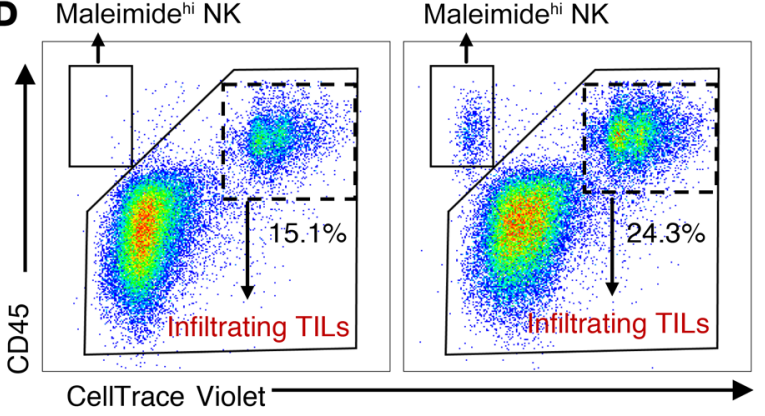

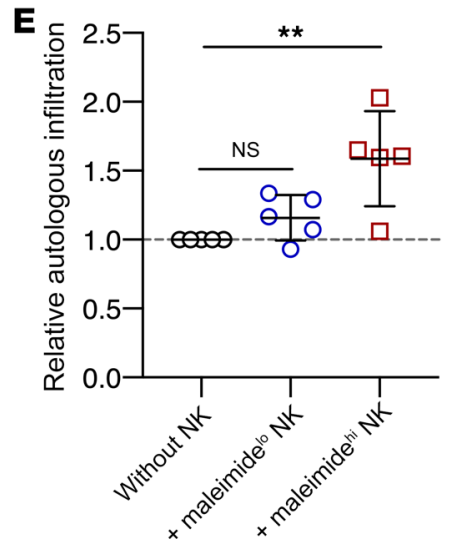

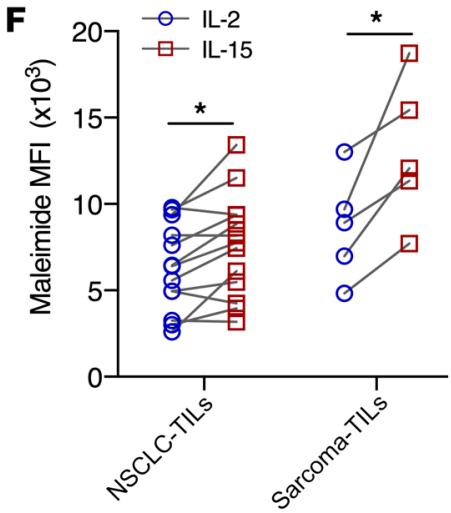

Figure 8. High surface thiol densities on tumor-infiltrating NK cells improve immune infiltration into tumors. (A) One of 10 representative histograms of maleimide staining on gated NK cells in different types of tissue samples isolated from patients with NSCLC. (B) MFI of maleimide staining comparing NK cells residing in different types of tissue samples from patients with NSCLC. Matched individual data points are connected with lines $(n=10)$. (C) Images showing t-SNE projections of single-cell RNA analysis from pooled NSCLC tumors $(n=7)$ and patients' peripheral blood $(n=6)$. Heatmap scale represents the NES for G0:0051353 and G0:0004791. (D) Representative FACS plots showing the gating strategy for analysis of autologous TIL infiltration into tumor spheres $(n=5)$. (E) Relative ratio of the percentage autologous infiltration normalized to the control (without NK) $(n=5)$. All data points are presented as the mean \pm SD. Kruskal-Wallis with Dunn's multiple-comparisons test was used to determine significance. (F) MFI of maleimide staining of NK cells in expanded TILs, comparing IL-2-primed and IL-15-primed cultures after 7 days ( $n=5$ sarcoma samples; $n=13$ NSCLC samples). Matched individual data points are connected with lines; all matching replicates are connected by lines. ${ }^{*} P<0.05$ and ${ }^{* *} P<0.01$, by Friedman's test (B) and mixed-model analysis with Holm-Šidák's multiple-comparisons test.

ies showed that thioredoxin activation is a sign of inflammation and is chemotactic to multiple immune cells (29). Yet, the current understanding of the thioredoxin pathway in immune cells, particularly NK cells, is not fully understood. We observed that the CD56 $6^{\text {bright }}$ subset of NK cells expressed higher levels of thioredoxin and persisted in lung tumors (data not shown). This could further explain how IL-15 agonist (ALT-803) priming enhances antitumor responses by the $\mathrm{CD} 56^{\text {bright }}$ subset of NK cells isolated from patients with multiple myeloma (30). Another recent study showed that the expansion of NK cells with K562 cells expressing 4-1BBL and membrane IL-15 upregulates the expression of thioredoxin and peroxiredoxin, which act as antioxidants that confer NK cell resistance to $\mathrm{H}_{2} \mathrm{O}_{2}$-mediated oxidative stress (31). Apart from the expression levels of thioredoxin and its inhibitory counterpart, TXNIP, we showed in NK cells that subcellular localization and shuttling of TXNIP has a crucial role in the regulation of thioredoxin activity. As also shown previously in pancreatic $\beta$ cells, the shuttling of TXNIP from the nucleus to the mitochon- 
dria within the cell is a response to oxidative stress. This in turn leads to ASK1 phosphorylation and cell death (32). In the immunology context, the thioredoxin/ASK1 axis was also found to regulate the selection and survival of double-positive thymocytes during T cell development (9). Although our previous study on the effects of IL-15 on NK cells revealed that mTOR activation acts as a main driver for enhanced immune functions, it is plausible that mTOR activation could be a regulator for the thioredoxin system (23). In fact, there was an evident link established between mTOR and the thioredoxin system. The inhibition of mTOR was found to induce cell death due to the inhibitory effect of TXNIP on the antioxidant pathway (33). By inhibiting mTOR with Torin-1, we observed both a reduction in thioredoxin activity and surface thiol density on the NK cells.

Another emphasis in the present study would be the importance of antioxidants in relation to immune infiltration and persistence. The thioredoxin system was previously found to explain the persistence of regulatory $\mathrm{T}$ cells in the microenvironment with high oxidative stress, which could be the link to the cell's surface thiol density (5). Similarly, thioredoxin is found to be upregulated in lamina propria $\mathrm{T}$ cells as compared with peripheral blood $\mathrm{T}$ cells (6). The present study indicates that antioxidants may not just contribute to sustain the killing capacity of NK cells but also equip these NK cells with a bystander role to protect other immune cells within proximity from ROS-mediated suppression. Given that NK cells residing in the tumor core express higher levels of surface thiol density, these NK cells not only have a reducing barrier that minimizes the accumulation of intracellular ROS but also possibly protects other TILs from ROS within the same microenvironment. Moreover, it was demonstrated that the induced production and secretion of thioredoxin in DCs could play a role in T cell activation (26). Even though it was not tested whether thioredoxin could be secreted by NK cells, we demonstrated that NK cells with high thiol density could promote the infiltration of $\mathrm{T}$ cells into autologous tumor spheres. Several studies also demonstrated how "helper" NK cells secrete an array of inflammatory cytokines that prime other immune cells to home to the tumor (34-36). Collectively, substantial evidence has elucidated how NK cells could turn a "cold," poorly infiltrated tumor into a "hot," inflammatory phenotype that is more compatible for immunotherapy.

Although the current mainstay of adoptive NK cell therapy involves the use IL-2 in expansion protocols, a number of clinical studies of solid tumors had reported no clinical benefits and low reactivity of these infused NK cells in the tumor $(19,37,38)$. The use of IL-15 in adoptive NK cell therapies should be further explored, as it was observed that endogenous IL-15 production was associated with the persistence of infused NK cells (39). In addition, IL-15 is currently incorporated into multispecific recombinant protein modalities as next-generation antibody-based therapies for cancers $(40,41)$. In the case of T cell expansion, IL-15-expanded $\mathrm{T}$ cells not only had more antioxidant effector molecules but also cytolytic molecules such as granzyme A and granzyme B (42).

The study of ROS and its effects on NK cells should also consider the involvement of tobacco smoking - a potent carcinogen with high ROS content (14). Early studies more than 2 decades ago found that cigarette smoking decreases NK cell frequency and activity $(43,44)$. On the other hand, conflicting reports demon- strated that cigarette smoking can stimulate NK cells to produce more IFN- $\gamma$ and activate other immune effector functions (45, 46). Though the underlying mechanism is not well understood, alveolar macrophages have been proposed to produce ROS to suppresses lung NK cell activity (47). In relation to IL-15, smoking was found to downregulate serum levels of IL-15 and its production by activated PBMCs $(48,49)$. In both our NSCLC cohort and TCGA public database, we found that tobacco smoking influences the amount of oxidative stress in tumor-infiltrating NK cells and that the expression of intratumoral IL-15 could improve the prognosis of patients who are either current smokers or had ceased smoking for less than 15 years. Also, when we excluded TCGA patients with NSCLC who did not have a cigarette pack-year history of smoking, $I L-15$ and the NK signature score also predicted favorable survival outcomes (data not shown). Although we observed no differences in NK cell gene signatures expressed by smokers versus nonsmokers (data not shown), it is nonetheless notable that the NK cell gene signature score predicted OS and PFI only in the smoker group.

In conclusion, our study exemplifies how environmental stress could influence the dynamic TME in cancer. We highlighted the crucial role of antioxidants in NK cells and how antioxidants can be modulated to improve NK cell-mediated therapies for solid tumors. Although IL-15 is already a clinically approved agent, future studies should also explore the incorporation of IL-15 into novel cell therapy products for better resistance against oxidative stress. Beyond the application of conventional immune checkpoint inhibitors, the understanding of cellular responses to oxidative stress is no doubt required to boost the efficacies of existing immunotherapeutic treatment regimes.

\section{Methods}

Cell culture. The NSCLC cell line H1299 and the chronic myelogenous leukemia cell line K562 (American Type Culture Collection [ATCC]) were maintained in RPMI medium (Life Technologies, Thermo Fisher Scientific) supplemented with $10 \%$ heat-inactivated FBS (Life Technologies, Thermo Fisher Scientific) and 1\% antibiotics (penicillin/ streptomycin). Patient-derived sarcoma primary cell lines (cases 1, 2, and 3 in Supplemental Table 2) were established from surgical resections and propagated with DMEM medium supplemented with GlutaMAX (Life Technologies, Thermo Fisher Scientific), 10\% heat-inactivated FBS, and $1 \%$ antibiotics (penicillin, streptomycin).

Tumor tissue processing. Tumor resections were processed using a Tumor Dissociation Kit (Miltenyi Biotec), and tumor cells were isolated with a negative selection-based Tumor Cell Isolation Kit (Miltenyi Biotec) according to the manufacturer's protocol. For FACS phenotyping, cell suspensions were washed with FACS buffer (PBS with 5\% FBS) and stained for flow cytometry after RBC lysis. For TIL proliferation assays, cells were labeled with CFSE (BioLegend) and then cultured for 7 days in DMEM/F12 medium supplemented with 10\% FBS and $2000 \mathrm{IU} / \mathrm{mL}$ IL-2 or IL-15 (PeproTech), in the presence or absence of catalase (200 IU/mL, MilliporeSigma).

Isolation of PBMCs, neutrophils, and NK cells. Human PBMCs were collected through Ficoll density gradient centrifugation (GE Healthcare). Primary NK cells were isolated by negative selection following the manufacture's protocol (Human NK Cell Isolation Kit, Miltenyi Biotec). Neutrophils from peripheral blood of healthy donors were enriched by the natural erythrocyte sedimentation method using dex- 
tran (MilliporeSigma). Isolated NK cells were cultured in X-VIVO 20 Medium (Lonza) supplemented with $10 \%$ heat-inactivated human $\mathrm{AB}$ serum for 48 hours, together with 300 IU/mL IL-2 or IL-15 for cytokine activation. Drugs or vehicle (DMSO) was added the day after NK cells were seeded in the plate overnight. Inhibitors used in this study included PX-12 (5 or $10 \mu \mathrm{M}$, SelleckChem), Torin-1 ( $1 \mu \mathrm{M}$, SelleckChem), $N$-ethylmaleimide (2.5 mM, MilliporeSigma), and recombinant thioredoxin-1 ( $5 \mathrm{mg} / \mathrm{mL}$, Thermo Fisher Scientific). For $\mathrm{H}_{2} \mathrm{O}_{2}$ treatment, activated NK cells were harvested and washed twice with PBS and then incubated for 1 hour in complete NK medium containing $5 \mu \mathrm{M}$ or $10 \mu \mathrm{M}$ hydrogen peroxide (MilliporeSigma).

For coculture experiments, autologous neutrophils and NK cells were obtained from fresh peripheral blood of a healthy donor and then seeded into 96-well plates at a 6:1 ratio with cytokine support and inhibitor treatment. LPS $(1 \mu \mathrm{g} / \mathrm{mL})$ was added to active neutrophils. Cells were thereafter cultured for 4 days before analysis by flow cytometry.

Expansion of TILs. After tumor dissociation, cell suspensions were cultured in AIM V Medium (Thermo Fisher Scientific) containing L-glutamine, streptomycin sulfate at $50 \mu \mathrm{g} / \mathrm{mL}$, and gentamicin sulfate at $10 \mu \mathrm{g} / \mathrm{mL}$, supplemented with $2.5 \%$ human $\mathrm{AB}$ serum and $3000 \mathrm{IU} /$ mL IL-15. After 7 days, irradiated PBMCs were added at a ratio of 200:1 as feeder cells. Cultures were maintained with $500 \mathrm{IU} / \mathrm{mL}$ IL-15 and a functional grade antibody against CD3 (Thermo Fisher Scientific). TILs were harvested 10 days later for subsequent experiments.

Cytotoxicity assay. A chromium $\left({ }^{51} \mathrm{Cr}\right)$ release assay was used to measure the cytolytic activity of activated NK cells. Briefly, K562 cells were harvested and labeled with ${ }^{51} \mathrm{Cr}$ (PerkinElmer) as target cells, cytokine-activated or sorted NK cells were used as effector cells. Cells were seeded in a 96-well V-bottom plate at the indicated $\mathrm{E} / \mathrm{T}$ ratio. The supernatants were carefully collected onto LUMA plates (PerkinElmer) after 6 and 16 hours of coculture. MicroBeta2 (PerkinElmer) was used to detected the radioactivity of the LUMA plate.

Flow cytometry and cell sorting. For surface marker staining, cells were incubated with fluorescence-conjugated antibodies for 20 minutes at $4^{\circ} \mathrm{C}$. For intracellular cytokine staining, NK cells were first stimulated with PMA and ionomycin or cocultured with K562 in the presence of BD GolgiStop and GolgiPlug for 4 hours, and then subjected to intracellular staining using a Cytofix/Cytoperm Kit (BD Biosciences). Ki-67 staining was performed with a Foxp3/Transcription Factor Staining Buffer Set (eBioscience). Cytofix/Phosflow Buffer (BD/ Biosciences) was used for phosphorylated S6 (p-S6)(Ser235/Ser236) staining. For Intracellular ROS, cells were stained using CellROX Deep Red Reagent and measured by flow cytometry following incubation for 30 minutes at $37^{\circ} \mathrm{C}(\mathrm{C} 10422$, Invitrogen, Thermo Fisher Scientific). Cell viability was analyzed by double staining with annexin $\mathrm{V}$ and 7-aminoactomycin $\mathrm{D}$ (7-AAD). Cell-surface thiol groups were measured by staining with $5 \mu \mathrm{M}$ Alexa Fluor $488 \mathrm{C}_{5}$ Maleimide (A10254, Thermo Fisher Scientific) for 15 minutes on ice. Stained cells were washed twice with FACS buffer and then acquired using NovoCyte (ACEA Biosciences). FlowJo (version 10) software (BD) was used to analyze flow cytometric data. The antibodies used for flow cytometry staining are listed in Supplemental Table 3. NK cells were sorted after 2 days of cytokine stimulation before harvesting and then stained with maleimide and Live/Dead cell marker (Thermo Fisher Scientific). Using BD FACSAria Fusion, NK cells with high and low thiol densities were sorted on the basis of gating of the top and bottom quintiles on maleimide histogram.
Western blotting. NK cells were harvested and lysed in RIPA lysis buffer (Cell Signaling Technology), supplemented with a protease and phosphatase inhibitor cocktail (Thermo Fisher Scientific). Cell lysates were centrifuged at maximum speed for 15 minutes and the supernatants collected. Lysates were boiled for 5 minutes at $96^{\circ} \mathrm{C}$ after addition of LDS Sample Buffer and Sample Reducing Agent (NuPAGE, Invitrogen, Thermo Fisher Scientific). Proteins were migrated onto $4 \%-12 \%$ Bis-Tris gradient gels (NuPAGE) and then transferred onto PVDF membranes. The membranes were blocked with $5 \% \mathrm{w} / \mathrm{v}$ skim milk before overnight incubation with anti-TXNIP Rabbit mAb (1:1000, ab188885, Abcam) at $4^{\circ} \mathrm{C}$, followed by incubation with anti-rabbit HRP (ab6721, Abcam) for 1 hour at room temperature. $\beta$-Actin (C4) HRP (47778, Santa Cruz Biotechnology) was used after washing the membrane with Restore Western Blot Stripping Buffer (Thermo Fisher Scientific) as an internal control. Protein bands were developed with Pierce ECL Western Blotting Substrate (32109, Thermo Fisher Scientific).

Thioredoxin activity assay. The thioredoxin activity of cytokine-primed NK cells was measured according to the manufacturer's instructions for the Fluorescent Thioredoxin Assay Kit (FkTRX-02-V2, IMCO). Briefly, $1 \times 10^{6} \mathrm{NK}$ cells were lysed in $200 \mu \mathrm{L}$ assay buffer by sonication. Cell lysates were incubated with thioredoxin reductase and NADPH in a 96 -well black micro titer plate at $37^{\circ} \mathrm{C}$ for 30 minutes. Following incubation, fluorescent substrate was added to each sample. The plate was immediately placed in a plate reader (Enspire, PerkinElmer), and fluorescence was recorded at $545 \mathrm{~nm}$ emission after 520 $\mathrm{nm}$ excitation for 60 minutes. Results were calculated and compared in accordance with the kit's instructions.

Sphere formation and infiltration. 3D sphere cultures were done with H1299 and 3 patient-derived sarcoma cell lines. For the H1299 cell line, $10^{4}$ cells were seeded in a Nunclon Sphera 96-well round-bottomed plate (Thermo Fisher Scientific) with complete RPMI medium, single spheres were formed after overnight culture. For the sarcoma cell lines, $10^{5}$ cells were seeded in low-attachment 12-well plates with DMEM/F12 medium supplemented with 10\% FBS and 2.5\% Matrigel. Spheres were formed within 3-5 days. Single cells in suspension and cells from loose clusters were removed before the addition of $3 \times 10^{5}$ fluorescence-labeled (either CFSE or CellTrace Violet) autologous TILs or sorted NK cells. Cocultures of spheres with immune cells were incubated for 48 hours before manual separation and washing of spheres from single-cell suspensions. Images of the sphere cocultures were acquired on an IncuCyte S3 System (Essen Bioscience, Sartorius). Isolated spheres were then digested with a Tumor Dissociation Kit (Miltenyi Biotec) to preserve surface markers for FACS analysis. Single-cell suspensions of isolated sphere and cells that were outside the spheres were collected separately for flow cytometry, and anti-CD45 antibody was added to identify immune cells and tumor cells.

Confocal imaging. For mitochondrial mass staining, NK cells were incubated with MitoTracker Deep Red FM (50 nM, Invitrogen, Thermo Fisher Scientific) in FBS-free RPMI medium for 30 minutes at $37^{\circ} \mathrm{C}$. The Foxp3/Transcription Factor Staining Buffer Set (eBioscience) was used to fix and permeabilize cells after staining of mitochondria. Cells were then stained with anti-TXNIP Rabbit mAb (ab188885, Abcam) followed by FITC-conjugated donkey anti-rabbit IgG antibody (406403, BioLegend). Stained cells were spun onto the slides using the Cytospin 2 (Shandon). Coverslips were mounted onto a Fluoroshield with DAPI Histology Mounting Medium (MilliporeSigma). Slide images were acquired with confocal LSM 700 (Zeiss). 
Images acquired with a $\times 40$ objective were processed and analyzed with CellProfiler for TXNIP object counts and fluorescence intensity. Images acquired using a $\times 63$ objective are presented as representative maximum-intensity projections.

RNA isolation and quantitative PCR. RNA was extracted from 5 $\times 10^{5}$ cytokine-primed NK cells following the manufacturer's guidelines for the RNeasy Micro Kit (QIAGEN). Complementary DNA was synthesized using a QuantiTect Reverse Transcription Kit (QIAGEN). Quantitative PCR was performed using Power SYBR Green PCR Master Mix (Thermo Fisher Scientific). The CFX96 Touch Real-Time PCR Detection System (Bio-Rad) was used to detect SYBR Green incorporation. Relative expression levels of target genes were calculated using the comparative Ct method, and TBP was used as an endogenous control. Primer sequences are shown in Supplemental Table 4.

Immunohistochemistry. Fresh tissues were fixed with neutral buffered formalin and embedded in paraffin and then cut into sections following regular histological procedures. After deparaffinization and rehydration, the sections were immersed in $10 \mathrm{mM}$ sodium citrate buffer ( $\mathrm{pH}$ 6.0) for heat-mediated antigen retrieval. Hydrogen peroxide solution and $4 \%$ normal goat serum was used to block endogenous peroxidase and nonspecific binding, respectively. The following primary antibodies were used: anti-NCAM1/CD56 (1:300, 14255-1-AP, Proteintech) and anti-8-OHdG (1:100, ab48508, Abcam). After overnight incubation with a primary antibody at $4^{\circ} \mathrm{C}$, an HRP-conjugated secondary antibody was used for 1 hour at room temperature. DAB was used as chromogen followed by counterstaining with hematoxylin. Sections were scanned and analyzed with CaseViewer software (3DHISTECH).

Gene expression analysis. Gene expression data analyzing polysome-associated mRNA in IL-2- and IL-15-activated NK cells by anota (analysis of translational activity) (50) were download from the NCBI's Gene Expression Omnibus (GEO) database (GEO GSE77808) (23). Generally applicable gene set enrichment analysis (GSEA) was used to identify enrichment of genes with functions annotated by the Gene Ontology Consortium using data for all genes as input. The GSEA analyses were performed using GSEA software (version 4.0.3). The predefined gene sets, which represent comprehensive biological processes, were obtained from the Molecular Signatures Database (MSigDB), version 7.0. We used the C5 GO set for GSEA analysis with the cutoff at a nominal value of $P<0.01$, and the maximum and minimum sizes for selection were 500 and 15 genes, respectively. The list of genes shows their ranking on the basis of their NES, which reflects the extent of statistically overrepresented gene sets in our data set of differentially expressed genes. Furthermore, to focus on the response to ROS, the GO term GO:0000302 was used to identify ROS-related genes. TBtools was used for heatmap visualization (51).

We assessed processed single-cell RNA-Seq data obtained from the NCBI's GEO database (GEO GSE127465) (52). Using SeqGeq (BD), we first normalized gene expression for dimension reduction using principal component analysis (PCA) followed by t-distributed stochastic neighbor embedding (t-SNE) analysis. The NK cytotoxicity gene set (GZMA, GZMB, GZMH, GZMM, GZMK, PRF1, NKG7, GNLY) was previously defined and used in another study (53). GO pathways from the MSigDB (GO:0051353 and GO:0004791) were imported into the software for enrichment analysis. The enrichment score was calculated by summing the various gene sets in SeqGeq. The geometric means of the enrichment score for defined cell populations were compared and tested for significance using GraphPad Prism 8 (GraphPad Software).

Processing of TCGA public data sets and gene signature application. Together with normalized gene expression data, raw data for OS and PFI, together with tobacco smoking history were exported from TCGA public database, accessed from the UCSC Xena browser (http://xena. ucsc.edu). Application of the NK cell gene signature for survival analysis (CD160, PRF1, KLRB1, NCR1, and NCR3) was previously done (22, 36). Correlation between 2 other published NK signatures and IL15 was done with custom $\mathrm{R}$ scripts and the corrplot package $(54,55)$. The oxidative stress gene signature was based on the sum of 42 genes correlated with prognosis in the NSCLC previously reported data set (24). The NSCLC TCGA cohort was then split into 2 groups on the basis of the patients' tobacco smoking history before downstream analysis.

Statistics. Using "survival” and survplot R packages, we performed Kaplan-Meier analysis, with the NK cell signature score or IL15 gene expression split into a binary (low/high) variable on the basis of the median value. Both OS and PFI were used as survival endpoints. All statistical tests in this study (Wilcoxon, Kruskal-Wallis, Mann-Whitney $U$, ANOVA, Friedman's, Spearman's rank correlation coefficient, and logrank) were performed using GraphPad Prism 8 (GraphPad Software). A $P$ value of less than 0.05 was considered statistically significant.

Study approval. This study was approved by the ethics committee of the Second Affiliated Hospital of Zhejiang University (IR2019001101) and the ethics review board of Karolinska Institutet (DNR:2013 1979-31). NSCLC surgical resections were collected at the Second Affiliated Hospital of Zhejiang University from 29 patients with a primary diagnosis of NSCLC. Sarcoma specimens and matched peripheral blood were obtained at Karolinska University Hospital from 15 patients with sarcoma. Written informed consent was provided by all patients before sample acquisition, in accordance with Declaration of Helsinki principles.

\section{Author contributions}

$\mathrm{AL}, \mathrm{YY}$, and SYN conceived and designed the project. YY and SYN performed most of the experiments and interpreted the data with the help of ZC, MG, AK and EA. SYN and YC performed the bioinformatics analysis and visualization. WC performed confocal imaging under the supervision of LH. FH provided human sarcoma samples. YW and HX participated in clinical lung cancer sample acquisition under the supervision of KW. YY and SYN composed all figures and wrote the manuscript with key editing by AL and further input from all authors. AL and KW supervised the project and reviewed the manuscript. The order of shared first authorship was determined by the respective contributions of these authors to this project: YY initiated the project and performed the majority of the experiments, and NSY contributed critical data collection and analysis during later stages and during the revision of the manuscript.

\section{Acknowledgments}

We thank Xingyuan Xu, Muyi Yang, and Zhiqing Fang for their technical support. This work was supported by the Swedish Cancer Society (no. CAN 2018/451), the Cancer Research Foundations of Radiumhemmet (grant 181183), The Sagen Foundation, and grants from the National Natural Science Foundation of China (grants 81902331 and 81871874). 
Address correspondence to: Andreas Lundqvist, Karolinska Institutet. Bioclinicum: J6:20 Theme Cancer, Akademiska stråket 1. S-17164 Solna, Sweden. Phone: 46.736422412; Email: andreas. lundqvist@ki.se. Or to: Kai Wang, Second Affiliated hospital, Zhejiang University, Jiefang Rd.88, Hangzhou, 310009, China. Phone: 86.13957158572; Email: kaiw@zju.edu.cn.
1. Aydin E, Johansson J, Nazir FH, Hellstrand K, Martner A. Role of NOX2-derived reactive oxygen species in NK cell-mediated control of murine melanoma metastasis. Cancer Immunol Res. 2017;5(9):804-811.

2. Nakamura K, Matsunaga K. Susceptibility of natural killer (NK) cells to reactive oxygen species (ROS) and their restoration by the mimics of superoxide dismutase (SOD). Cancer Biother Radiopharm. 1998;13(4):275-290.

3. Stiff A, et al. Nitric oxide production by myeloid-derived suppressor cells plays a role in impairing Fc receptor-mediated natural killer cell function. Clin Cancer Res. 2018;24(8):1891-1904.

4. Valko M, Rhodes CJ, Moncol J, Izakovic M, Mazur $M$. Free radicals, metals and antioxidants in oxidative stress-induced cancer. Chem Biol Interact. 2006;160(1):1-40.

5. Mougiakakos D, Johansson CC, Jitschin R, Böttcher M, Kiessling R. Increased thioredoxin-1 production in human naturally occurring regulatory $\mathrm{T}$ cells confers enhanced tolerance to oxidative stress. Blood. 2011;117(3):857-861.

6. Sido B, Giese T, Autschbach F, Lasitschka F, Braunstein J, Meuer SC. Potential role of thioredoxin in immune responses in intestinal lamina propria T lymphocytes. Eur JImmunol. 2005;35(2):408-417.

7. Collet JF, Messens J. Structure, function, and mechanism of thioredoxin proteins. Antioxid Redox Signal. 2010;13(8):1205-1216.

8. Holmgren A. Thioredoxin and glutaredoxin systems. J Biol Chem. 1989;264(24):13963-13966.

9. Jin R, et al. Trx1/TrxR1 system regulates postselected DP thymocytes survival by modulating ASK1-JNK/p38 MAPK activities. Immunol Cell Biol. 2015;93(8):744-752.

10. Kim SH, Oh J, Choi JY, Jang JY, Kang MW, Lee CE. Identification of human thioredoxin as a novel IFN-gamma-induced factor: mechanism of induction and its role in cytokine production. BMC Immunol. 2008;9:64.

11. Chakraborty P, et al. Thioredoxin- 1 improves the immunometabolic phenotype of antitumor $\mathrm{T}$ cells. J Biol Chem. 2019;294(23):9198-9212.

12. Carter BD, Freedman ND, Jacobs EJ. Smoking and mortality--beyond established causes. $N$ Engl JMed. 2015;372(22):2170.

13. Pfeifer GP, Denissenko MF, Olivier M, Tretyakova N, Hecht SS, Hainaut P. Tobacco smoke carcinogens, DNA damage and p53 mutations in smoking-associated cancers. Oncogene. 2002;21(48):7435-7451.

14. Brody JS, Spira A. State of the art. Chronic obstructive pulmonary disease, inflammation, and lung cancer. Proc Am Thorac Soc. 2006;3(6):535-537.

15. Rizvi NA, et al. Cancer immunology. Mutational landscape determines sensitivity to PD-1 blockade in non-small cell lung cancer. Science. 2015;348(6230):124-128.

16. Gettinger S, et al. Impaired HLA class I antigen processing and presentation as a mechanism of acquired resistance to immune checkpoint inhibitors in lung cancer. Cancer Discov. 2017;7(12):1420-1435.

17. Kärre K. Natural killer cell recognition of missing self. Nat Immunol. 2008;9(5):477-480.

18. Vivier E, Tomasello E, Baratin M, Walzer T, Ugolini S. Functions of natural killer cells. Nat Immunol. 2008;9(5):503-510.

19. Oh S, Lee JH, Kwack K, Choi SW. Natural killer cell therapy: a new treatment paradigm for solid tumors. Cancers (Basel). 2019;11(10):E1534.

20. López-Soto A, Gonzalez S, Smyth MJ, Galluzzi L. Control of metastasis by NK cells. Cancer Cell. 2017;32(2):135-154.

21. Crome SQ, Lang PA, Lang KS, Ohashi PS. Natural killer cells regulate diverse $\mathrm{T}$ cell responses. Trends Immunol. 2013;34(7):342-349.

22. Neo SY, et al. CD73 immune checkpoint defines regulatory NK cells within the tumor microenvironment. J Clin Invest. 2020;130(3):1185-1198.

23. Mao Y, et al. IL-15 activates mTOR and primes stress-activated gene expression leading to prolonged antitumor capacity of NK cells. Blood. 2016;128(11):1475-1489.

24. Leone A, Roca MS, Ciardiello C, Costantini S, Budillon A. Oxidative stress gene expression profile correlates with cancer patient poor prognosis: identification of crucial pathways might aelect novel therapeutic approaches. Oxid Med Cell Longev. 2017;2017:2597581.

25. Werlenius $\mathrm{O}$, et al. Reactive oxygen species induced by therapeutic CD2O antibodies inhibit natural killer cell-mediated antibody-dependent cellular cytotoxicity against primary CLL cells. Oncotarget. 2016;7(22):32046-32053.

26. Angelini G, et al. Antigen-presenting dendritic cells provide the reducing extracellular microenvironment required for $\mathrm{T}$ lymphocyte activation. Proc Natl Acad Sci USA. 2002;99(3):1491-1496.

27. Castellani P, Angelini G, Delfino L, Matucci A, Rubartelli A. The thiol redox state of lymphoid organs is modified by immunization: role of different immune cell populations. Eur JImmunol. 2008;38(9):2419-2425.

28. Levring TB, et al. Tumor necrosis factor induces rapid down-regulation of TXNIP in human T cells. Sci Rep. 2019;9(1):16725.

29. Bertini R, et al. Thioredoxin, a redox enzyme released in infection and inflammation, is a unique chemoattractant for neutrophils, monocytes, and T cells. JExp Med.1999;189(11):1783-1789.

30. Wagner JA, et al. CD56bright NK cells exhibit potent antitumor responses following IL-15 priming. J Clin Invest. 2017;127(11):4042-4058.

31. Mimura K, et al. Upregulation of thioredoxin-1 in activated human NK cells confers increased tolerance to oxidative stress. Cancer Immunol Immunother. 2017;66(5):605-613.

32. Saxena G, Chen J, Shalev A. Intracellular shuttling and mitochondrial function of thioredoxin-interacting protein. J Biol Chem.
2010;285(6):3997-4005.

33. Malone CF, et al. mTOR and HDAC inhibitors converge on the TXNIP/thioredoxin pathway to cause catastrophic oxidative stress and regression of RAS-driven tumors. Cancer Discov. 2017;7(12):1450-1463.

34. Sedlacek AL, Kinner-Bibeau LB, Binder RJ. Phenotypically distinct helper NK cells are required for gp96-mediated anti-tumor immunity. Sci Rep. 2016;6:29889.

35. Wong JL, Berk E, Edwards RP, Kalinski P. IL-18primed helper NK cells collaborate with dendritic cells to promote recruitment of effector $\mathrm{CD}^{+} \mathrm{T}$ cells to the tumor microenvironment. Cancer Res. 2013;73(15):4653-4662.

36. Böttcher JP, et al. NK cells stimulate recruitment of cDC1 into the tumor microenvironment promoting cancer immune control. Cell. 2018;172(5):1022-1037.e14.

37. Bachanova V, et al. Clearance of acute myeloid leukemia by haploidentical natural killer cells is improved using IL-2 diphtheria toxin fusion protein. Blood. 2014;123(25):3855-3863.

38. Liu E, et al. Cord blood NK cells engineered to express IL-15 and a CD19-targeted CAR show long-term persistence and potent antitumor activity. Leukemia. 2018;32(2):520-531.

39. Miller JS, et al. Successful adoptive transfer and in vivo expansion of human haploidentical NK cells in patients with cancer. Blood. 2005;105(8):3051-3057.

40. Vallera DA, et al. IL15 trispecific killer engagers (TriKE) make natural killer cells specific to $\mathrm{CD}_{3}{ }^{+}$targets while also inducing persistence, in vivo expansion, and enhanced function. Clin Cancer Res. 2016;22(14):3440-3450.

41. Kim PS, et al. IL-15 superagonist/IL-15R $\alpha$ Sushi-Fc fusion complex (IL-15SA/IL-15R $\alpha \mathrm{Su}-\mathrm{Fc}$; ALT-803) markedly enhances specific subpopulations of NK and memory CD8+ T cells, and mediates potent anti-tumor activity against murine breast and colon carcinomas. Oncotarget. 2016;7(13):16130-16145.

42. Kaur N, et al. T cells expanded in presence of IL-15 exhibit increased antioxidant capacity and innate effector molecules. Cytokine. 2011;55(2):307-317.

43. Phillips B, Marshall ME, Brown S, Thompson JS. Effect of smoking on human natural killer cell activity. Cancer. 1985;56(12):2789-2792.

44. Tollerud DJ, et al. Association of cigarette smoking with decreased numbers of circulating natural killer cells. Am Rev Respir Dis. 1989;139(1):194-198.

45. Motz GT, et al. Chronic cigarette smoke exposure primes NK cell activation in a mouse model of chronic obstructive pulmonary disease. J Immunol. 2010;184(8):4460-4469.

46. Newman LS, Kreiss K, Campbell PA. Natural killer cell tumoricidal activity in cigarette smokers and in silicotics. Clin Immunol Immunopathol. 1991;60(3):399-411. 
47. Takeuchi M, et al. Inhibition of lung natural killer cell activity by smoking: the role of alveolar macrophages. Respiration. 2001;68(3):262-267.

48. Mian MF, Pek EA, Mossman KL, Stämpfli MR, Ashkar AA. Exposure to cigarette smoke suppresses IL-15 generation and its regulatory NK cell functions in poly I:C-augmented human PBMCs. Mol Immunol. 2009;46(15):3108-3116.

49. Shiels MS, et al. Cigarette smoking and variations in systemic immune and inflammation markers. J Natl Cancer Inst. 2014;106(11):dju294.
50. Larsson O, Sonenberg N, Nadon R. anota: Analysis of differential translation in genome-wide studies. Bioinformatics. 2011;27(10):1440-1441.

51. Chen C, et al. TBtools: an integrative toolkit developed for interactive analyses of big biological data [published online June 23, 2020]. Mol Plant. https://doi.org/10.1016/ j.molp.2020.06.009.

52. Zilionis R, et al. Single-cell transcriptomics of human and mouse lung cancers reveals conserved myeloid populations across individuals and spe- cies. Immunity. 2019;50(5):1317-1334.e10.

53. de Andrade LF, et al. Discovery of specialized NK cell populations infiltrating human melanoma metastases. JCI Insight. 2019;4(23):133103.

54. Zheng X, et al. Mitochondrial fragmentation limits NK cell-based tumor immunosurveillance. Nat Immunol. 2019;20(12):1656-1667.

55. Cursons J, et al. A gene signature predicting natural killer cell infiltration and improved survival in melanoma patients. Cancer Immunol Res. 2019;7(7):1162-1174. 